\title{
KOLMOGOROV WIDTHS AND APPROXIMATION NUMBERS OF SOBOLEV CLASSES WITH SINGULAR WEIGHTS
}

\author{
A. A. VASIL'EVA
}

\begin{abstract}
The Kolmogorov widths of weighted Sobolev classes in weighted $L_{q^{-}}$ spaces and the approximation numbers of the corresponding embedding operators are estimated. The case where the weights affect the asymptotics is considered.
\end{abstract}

\section{$\S 1$. INTRODUCTION}

Our aim in this paper is to estimate the values of Kolmogorov widths for weighted Sobolev classes $W_{p, g}^{r}\left[0, e^{-1}\right]$ in the spaces $L_{q, v}\left[0, e^{-1}\right]$ for a special class of weights.

We denote by $\mathbb{N}, \mathbb{Z}, \mathbb{Z}_{+}, \mathbb{R}, \mathbb{R}_{+}$the sets of natural numbers, integers, nonnegative integers, reals, and nonnegative reals, respectively.

For a measurable set $E \subset \mathbb{R}$, we denote by $L_{0}(E)$ the space of measurable real-valued functions on $E$ and by $L_{0}\left(E, \mathbb{R}_{+}\right)$the set of nonnegative measurable functions on $E$. With any $f \in L_{0}(E)$ we associate the quantity $\|f\|_{q} \in \mathbb{R}_{+} \cup\{\infty\}(0<q \leq \infty)$, where

$$
\|f\|_{q}=\|f\|_{L_{q}(E)}=\left\{\begin{array}{lll}
\left(\int_{E}|f(x)|^{q} d x\right)^{1 / q} & \text { if } q<\infty, \\
{\operatorname{ess} \sup _{x \in E}|f(x)|} \text { if } q=\infty .
\end{array}\right.
$$

If $\|f\|_{L_{q}(E)}<\infty$, then we write $f \in L_{q}(E)$. For $f \in L_{0}(E)$ and $v \in L_{0}\left(E, \mathbb{R}_{+}\right)$, $1 \leq q \leq \infty$, we put

$$
\|f\|_{q, v}=\|f\|_{L_{q, v}(E)}:=\|v f\|_{L_{q}(E)} \in \mathbb{R}_{+} \cup\{\infty\} .
$$

Let $L_{q, v}(E)$ be the space of all $f \in L_{0}(E)$ such that $\|f\|_{q, v}<\infty$, and let $L_{0, q, v}=$ $L_{0, q, v}(E)$ be the space $L_{0}(E)$ endowed with the seminorm $\|\cdot\|_{q, v}$.

We denote by $A C[a, b]$ the set of absolutely continuous functions on $[a, b]$.

For $1 \leq p \leq \infty, r \in \mathbb{N}$, and $g \in L_{0}\left([a, b], \mathbb{R}_{+}\right)$, we define a Sobolev class with the weight $g$ on $[a, b]$ by

$$
W_{p, g}^{r}[a, b]=\left\{f:[a, b] \rightarrow \mathbb{R} \mid f^{(r-1)} \in A C[a, b],\left\|\frac{f^{(r)}}{g}\right\|_{L_{p}[a, b]} \leq 1\right\}
$$

here if $g(x)=0$ on a set $E \subset[a, b]$ with positive measure, then for $f \in W_{p, g}^{r}[a, b]$ we set $\left\|\frac{f^{(r)}}{g}\right\|_{L_{p}[a, b]}=\left\|\frac{f^{(r)}}{g}\right\|_{L_{p}([a, b] \backslash E)}$. In particular,

$$
W_{\infty, g}^{r}[a, b]=\left\{f:[a, b] \rightarrow \mathbb{R}\left|f^{(r-1)} \in A C[a, b],\right| f^{(r)}(t) \mid \leq g(t) \text { a.e. }\right\} .
$$

Let $X, Y$ be sets, and let $f_{1}, f_{2}: X \times Y \rightarrow \mathbb{R}_{+}$. We write $f_{1}(x, y) \underset{y}{\lesssim} f_{2}(x, y)$

$\left(f_{2}(x, y) \underset{y}{\gtrsim} f_{1}(x, y)\right.$, or $f_{1}(x, y) \stackrel{x}{\lesssim} f_{2}(x, y)$, or $\left.f_{2}(x, y) \stackrel{x}{\gtrsim} f_{1}(x, y)\right)$ if there exists a positive

2010 Mathematics Subject Classification. Primary 41A46.

Key words and phrases. Weighted Sobolev classes, Kolmogorov widths, approximation numbers.

Supported by RFBR (grants nos. 09-01-00093 and 10-01-00442). 
function $c: Y \rightarrow \mathbb{R}$ such that $f_{1}(x, y) \leq c(y) f_{2}(x, y)$ for any $x \in X ; f_{1}(x, y) \underset{y}{\breve{y}} f_{2}(x, y)$ (or $\left.f_{1}(x, y) \stackrel{x}{\approx} f_{2}(x, y)\right)$ if $f_{1}(x, y) \underset{y}{\lesssim} f_{2}(x, y) \underset{y}{\underset{y}{\lessgtr}} f_{1}(x, y)$.

Throughout, we use the notation $p^{\prime}=\frac{p}{p-1}, q^{\prime}=\frac{q}{q-1}, \varkappa=\frac{1}{r+\frac{1}{q}-\frac{1}{p}}$.

Let $X$ be a linear space with a seminorm (which may also take infinite values), let $n \in \mathbb{Z}_{+}$, and let $\mathcal{L}_{n}(X)$ be the set of subspaces in $X$ of dimension at most $n$. We denote by $L(X, Y)$ the space of continuous linear operators from $X$ into a normed space $Y$ and by rk $A$ the dimension of the image of an operator $A: X \rightarrow Y$. By the Kolmogorov $n$-width of a set $M \subset X$ in the space $X$ we mean the quantity

$$
d_{n}(M, X)=\inf _{L \in \mathcal{L}_{n}(X)} \sup _{x \in M} \inf _{y \in L}\|x-y\|_{X},
$$

and by the linear $n$-width (in the case of a normed space) we mean the quantity

$$
\lambda_{n}(M, X)=\inf _{\substack{A \in L(X, X) \\ \operatorname{rk} A \leq n}} \sup _{x \in M}\|x-A x\|_{X} .
$$

The approximation numbers of $A \in L(X, Y)$ are defined by

$$
\mathcal{A}_{n}(A)=\inf \left\{\left\|A-A_{n}\right\|_{X \rightarrow Y}: \operatorname{rk} A_{n} \leq n\right\} .
$$

If $A$ is the operator of embedding of $X$ in $Y$, then we denote $\mathcal{A}_{n}(A)=\mathcal{A}_{n}\left(B_{X}, Y\right)$. If $A$ is compact, then Heinrich's results in [1] imply that

$$
\mathcal{A}_{n}(M, Y)=\lambda_{n}(A(M), Y) \text {. }
$$

In the 1960-1970s, problems concerning the values of the widths of function classes in $L_{q}$ and of the finite-dimensional balls $B_{p}^{n}$ in $l_{q}^{n}$ were investigated (see [2, 3, 4, 5, 6, 7, 8, 9, 10, 11] and also [12, 13, 14]). Here $l_{q}^{n}(1 \leq q \leq \infty)$ is the space $\mathbb{R}^{n}$ with the norm

$$
\left\|\left(x_{1}, \ldots, x_{n}\right)\right\|_{q} \equiv\left\|\left(x_{1}, \ldots, x_{n}\right)\right\|_{l_{q}^{n}}=\left\{\begin{array}{lll}
\left(\left|x_{1}\right|^{q}+\cdots+\left|x_{n}\right|^{q}\right)^{1 / q} & \text { if } & q<\infty \\
\max \left\{\left|x_{1}\right|, \ldots,\left|x_{n}\right|\right\} & \text { if } & q=\infty,
\end{array}\right.
$$

and $B_{p}^{n}$ is the unit ball in $l_{p}^{n}$. In the case where $p \geq q$, the exact values for the widths of finite-dimensional balls were found, and the widths of Sobolev classes were shown to coincide with the reciprocals to the spectral numbers for a certain differential equation (in general, this equation is nonlinear). Moreover, the asymptotics of the widths was found. For $1<p<q<\infty$ (and in some cases for $p=1$ or $q=\infty$ ), the orders for the widths of finite-dimensional balls and Sobolev classes were found. These results will be stated in the next section.

In several papers (see, e.g., [15, 16, 17, 18, 19, 20]) sufficient conditions on weight functions were found for which the Kolmogorov widths or the approximation numbers of embedding operators for weighted Sobolev classes have the same orders as in the nonweighted case.

In [21, the definition of continuous embedding of $W_{p, g}^{r}[a, b]$ in $L_{q, v}[a, b]$ was given in terms of widths. Then a criterion for the existence of a continuous embedding was obtained and the concept of a reduced Sobolev class was introduced. The continuous embedding operator for a reduced Sobolev class is constructed in a natural way. Also, it was shown that, in many cases, the asymptotic behavior of widths does not depend on the choice of a reduced Sobolev class.

We say that there exists a continuous embedding of $W_{p, g}^{r}[a, b]$ in $L_{q, v}[a, b]$ (see [21]) if there exists $n \in \mathbb{N}$ such that

$$
d_{n}\left(W_{p, g}^{r}[a, b], L_{0, q, v}[a, b]\right)<\infty .
$$

Now we define weighted Riemann-Liouville operators and their compositions. 
Let $-\infty<a<b<+\infty$, and let $g, v \in L_{0}\left([a, b], \mathbb{R}_{+}\right)$. We define operators $I_{r, g, v, a}$ : $L_{p}[a, b] \rightarrow L_{q}[a, b]$ and $\widetilde{I}_{r, g, v, b}: L_{p}[a, b] \rightarrow L_{q}[a, b]$ by

$$
\begin{aligned}
& \left(I_{r, g, v, a} f\right)(x)=v(x) \int_{a}^{x}(x-t)^{r-1} g(t) f(t) d t, \\
& \left(\widetilde{I}_{r, g, v, b} f\right)(x)=v(x) \int_{x}^{b}(t-x)^{r-1} g(t) f(t) d t
\end{aligned}
$$

(in general, these operators are unbounded). Denote $\mathcal{L}_{g}=\left\{f \in L_{0}[a, b]: f g \in L_{1}[a, b]\right\}$. Let $I_{r, g, v, a}$ and $\tilde{I}_{r, g, v, b}: \mathcal{L}_{g} \rightarrow L_{0}[a, b]$ be defined by (1.2). For each $k=1, \ldots, r-1$, we put

$$
\begin{aligned}
I_{r, g, v}^{a, b, k} & =\widetilde{I}_{k, 1, v, b} \circ I_{r-k, g, 1, a}, & \widetilde{I}_{r, g, v}^{a, b, k} & =I_{k, 1, v, a} \circ \widetilde{I}_{r-k, g, 1, b}, \\
I_{r, g, v}^{a, b, 0} & =\widetilde{I}_{r, g, v}^{a, b, r}=I_{r, g, v, a}, & I_{r, g, v}^{a, b, r} & =\widetilde{I}_{r, g, v}^{a, b, 0}=\widetilde{I}_{r, g, v, b} .
\end{aligned}
$$

Note that

$$
\begin{aligned}
& \left\{\frac{1}{(k-1) !(r-k-1) !} I_{r, g, 1}^{a, b, k} \psi:\|\psi\|_{L_{p}[a, b]} \leq 1\right\} \\
& =\left\{f \in W_{p, g}^{r}[a, b]: \begin{array}{l}
f^{(j)}(b)=0 \text { for } 0 \leq j \leq k-1, \\
f^{(j)}(a)=0 \text { for } k \leq j \leq r-1
\end{array}\right\}, \\
& \left\{\frac{1}{(k-1) !(r-k-1) !} \widetilde{I}_{r, g, 1}^{a, b, k} \psi:\|\psi\|_{L_{p}[a, b]} \leq 1\right\} \\
& =\left\{f \in W_{p, g}^{r}[a, b]: \begin{array}{l}
f^{(j)}(a)=0 \text { for } 0 \leq j \leq k-1, \\
f^{(j)}(b)=0 \text { for } k \leq j \leq r-1
\end{array}\right\}
\end{aligned}
$$

(throughout, we assume that $(-1) !=1$ ). The set defined by (1.3) (or by (1.4)) is bounded in $L_{q, v}[a, b]$ if and only if the operator $I_{r, g, v}^{a, b, k}: L_{p}[a, b] \rightarrow L_{q}[a, b]\left(\right.$ or $\widetilde{I}_{r, g, v}^{a, b, k}: L_{p}[a, b] \rightarrow$ $L_{q}[a, b]$, respectively) is bounded.

A criterion for the boundedness of the Riemann-Liouville operators from $L_{p}[a, b]$ to $L_{q}[a, b]$ and estimates for their norms were obtained by Stepanov [22, 23, 24, 25, 26, and a criterion for the boundedness of $\widetilde{I}_{r, g, v}^{a, b, k}$ for $k=1, \ldots, r-1$ was obtained by Heinig and Kufner [27] (their results will be formulated in the next section).

In 21], it was shown that if $g \in L_{p^{\prime}}[a+\delta, b]$ and $v \in L_{q}[a+\delta, b]$ for any $\delta \in(0, b-a)$, then the existence of a continuous embedding is equivalent to the existence of $k \in\{0, \ldots, r\}$ such that the operator $\widetilde{I}_{r, g, v}^{a, b, k}: L_{p}[a, b] \rightarrow L_{q}[a, b]$ is bounded. If such $k$ exists, then we define the reduced Sobolev class by

$$
\widehat{W}_{p, g}^{r}[a, b]=\left\{\frac{1}{(k-1) !(r-k-1) !} \widetilde{I}_{r, g, 1}^{a, b, k} \psi:\|\psi\|_{L_{p}[a, b]} \leq 1\right\} .
$$

This subset of $L_{q, v}[a, b]$ is bounded and absolutely convex. Therefore, the Kolmogorov widths of this class, the embedding operator of the linear span of $\widehat{W}_{p, g}^{r}[a, b]$, and the approximation numbers of this operator are well defined (the class $W_{p, g}^{r}[a, b]$ may fail to be included in the space $\left.L_{q, v}[a, b]\right)$.

If $g(x)=x^{-\beta_{g}}$ and $v(x)=x^{-\beta_{v}}$, then for $\beta_{g}+\beta_{v}<r+\frac{1}{q}-\frac{1}{p}$ there exists a continuous (and even compact) embedding of $W_{p, g}^{r}[0, c]$ in $L_{q, v}[0, c], c>0$. In the case where $\beta_{g}+\beta_{v}>r+\frac{1}{q}-\frac{1}{p}$, no continuous embedding can exist.

In this paper we consider weights that are close to power weights in a neighborhood of the origin; the sum of exponents will be equal to the critical exponent $-r-\frac{1}{q}+\frac{1}{p}$. We formulate the main results. 
Definition 1. We say that a function $\varphi:[1,+\infty) \rightarrow(0,+\infty)$ satisfies condition (\#) if for any $\varepsilon>0$ there exist bounded functions $h_{\varepsilon, \pm}:[1,+\infty) \rightarrow \mathbb{R}$, a monotone increasing function $\psi_{\varepsilon,+}:[1,+\infty) \rightarrow \mathbb{R}$, and a monotone decreasing function $\psi_{\varepsilon,-}:[1,+\infty) \rightarrow \mathbb{R}$ such that $\ln \varphi(t) \pm \varepsilon t=\psi_{\varepsilon, \pm}(t)+h_{\varepsilon, \pm}(t)$; in this case, we denote

$$
c_{\varphi}(\varepsilon)=\max \left\{\left\|h_{\varepsilon,+}\right\|_{L_{\infty}[1,+\infty)},\left\|h_{\varepsilon,-}\right\|_{L_{\infty}[1,+\infty)}\right\} .
$$

For $t \in \mathbb{R}$, we set $(t)_{+}=\max \{t, 0\}$.

Theorem 1. Let $r \in \mathbb{N}$, let $1<p \leq+\infty, 1 \leq q<+\infty$, let $\beta_{g}, \beta_{v} \in \mathbb{R}, \beta_{g}+\beta_{v}=r+\frac{1}{q}-\frac{1}{p}$, $\beta_{v} \neq \frac{1}{q}+k, k=0,1, \ldots, r-1$, and let $g(x)=x^{-\beta_{g}} \varphi_{g}(|\ln x|), v(x)=x^{-\beta_{v}} \varphi_{v}(|\ln x|)$. Suppose that functions $\varphi_{g}$ and $\varphi_{v}$ satisfy (\#) and the following conditions:

(1) we have

$$
\left\|\varphi_{g} \varphi_{v}\right\|_{L_{s}[n, \infty)} n^{r-\left(\frac{1}{p}-\frac{1}{q}\right)}+\stackrel{n}{\lesssim}\left\|\varphi_{g} \varphi_{v}\right\|_{L_{\varkappa}[1, n]},
$$

where $\frac{1}{s}=\left(\frac{1}{q}-\frac{1}{p}\right)_{+}$;

(2) if $p<q$ and $q>2$, then there exist numbers $\alpha>\frac{1}{q} \min \left\{\frac{\frac{1}{p}-\frac{1}{q}}{\frac{1}{2}-\frac{1}{q}}, 1\right\}$ and $M>0$ such that

$$
\varphi_{g}(c y) \varphi_{v}(c y) \leq M c^{-\alpha} \varphi_{g}(y) \varphi_{v}(y) \text { for any } c \geq 1, \quad y \geq 1 .
$$

Then

$$
d_{n}\left(\widehat{W}_{p, g}^{r}\left[0, e^{-1}\right], L_{q, v}\left[0, e^{-1}\right]\right) \stackrel{n}{ }\left\|\varphi_{g} \varphi_{v}\right\|_{L_{\varkappa}[1, n]} d_{n}\left(W_{p}^{r}[0,1], L_{q}[0,1]\right) .
$$

As an example, we consider the functions $\varphi_{g}(y)=y^{-\alpha_{g}} \rho_{g}(y), \varphi_{v}(y)=y^{-\alpha_{v}} \rho_{v}(y)$, where $\alpha:=\alpha_{g}+\alpha_{v}>\alpha_{p q}$,

$$
\alpha_{p q}=\left\{\begin{array}{lll}
\frac{1}{q}-\frac{1}{p} & \text { if } q<p, \\
0 & \text { if } p \leq q \leq 2, \\
\frac{1}{q} & \text { if } p \leq 2<q, \\
\frac{1}{q} \frac{1}{\frac{p}{2}-\frac{1}{q}} \frac{1}{q} & \text { if } 2 \leq p \leq q,
\end{array}\right.
$$

$\alpha \neq r+\frac{1}{q}-\frac{1}{p}$, and the functions $\rho_{g}, \rho_{v}:[1, \infty) \rightarrow(0, \infty)$ are absolutely continuous on each finite segment and satisfy

$$
\frac{y \rho_{g}^{\prime}(y)}{\rho_{g}(y)} \rightarrow 0 \text { and } \frac{y \rho_{v}^{\prime}(y)}{\rho_{v}(y)} \rightarrow 0, \quad y \rightarrow \infty .
$$

If $p<q$ and $q>2$, then (1.6) is fulfilled for $\alpha>\frac{1}{q} \min \left\{\frac{\frac{1}{p}-\frac{1}{q}}{\frac{1}{2}-\frac{1}{q}}, 1\right\}$. In the case of the opposite inequality (if it is strict), it is also possible to obtain the orders of the widths.

Theorem 2. Suppose $r \in \mathbb{N}, p>1, \max \{p, 2\}<q<+\infty$ and $0<\alpha<\frac{1}{q} \min \left\{\frac{\frac{1}{p}-\frac{1}{q}}{\frac{1}{2}-\frac{1}{q}}, 1\right\}$, $\varphi_{g}(y)=y^{-\alpha_{g}} \rho_{g}(y), \varphi_{v}(y)=y^{-\alpha_{v}} \rho_{v}(y), \alpha_{g}+\alpha_{v}=\alpha$, and $\rho_{g}, \rho_{v}$ satisfy (1.9). Let $g(x)=x^{-\beta_{g}} \varphi_{g}(|\ln x|), v(x)=x^{-\beta_{v}} \varphi_{v}(|\ln x|), x \in\left(0, e^{-1}\right]$, and let $\beta_{g}+\beta_{v}=r+\frac{1}{q}-\frac{1}{p}$, $\beta_{v} \neq \frac{1}{q}+k, k=0, \ldots, r-1$. Denote $\rho(y)=\rho_{g}(y) \rho_{v}(y)$. Then

$$
d_{n}\left(\widehat{W}_{p, g}^{r}\left[0, e^{-1}\right], L_{q, v}\left[0, e^{-1}\right]\right) \stackrel{n}{`} n^{-\frac{\alpha q}{2}} \rho\left(n^{q / 2}\right) .
$$

Similar assertions on the orders of approximation numbers look like this. In Theorem 1, condition 2 is replaced by

- if $1<p<2<q<\infty$, then there exist numbers $\alpha>\max \left\{\frac{1}{q}, \frac{1}{p^{\prime}}\right\}$ and $M>0$ such that (1.6) is true. 
In this case

$$
\begin{aligned}
& \lambda_{n}\left(\widehat{W}_{p, g}^{r}\left[0, e^{-1}\right], L_{q, v}\left[0, e^{-1}\right]\right) \stackrel{[1.1}{=} \mathcal{A}_{n}\left(\widehat{W}_{p, g}^{r}\left[0, e^{-1}\right], L_{q, v}\left[0, e^{-1}\right]\right) \\
& \stackrel{n}{=}\left\|\varphi_{g} \varphi_{v}\right\|_{L_{\varkappa}[1, n]} \lambda_{n}\left(W_{p}^{r}[0,1], L_{q}[0,1]\right) .
\end{aligned}
$$

In Theorem 2, the conditions on $p, q, \alpha$ are rewritten as follows: $1<p<2<q<\infty$, $0<\alpha<\max \left\{\frac{1}{q}, \frac{1}{p^{\prime}}\right\}$. In this case,

$$
\begin{aligned}
\lambda_{n}\left(\widehat{W}_{p, g}^{r}\left[0, e^{-1}\right], L_{q, v}\left[0, e^{-1}\right]\right) & \stackrel{[1.1}{=} \mathcal{A}_{n}\left(\widehat{W}_{p, g}^{r}\left[0, e^{-1}\right], L_{q, v}\left[0, e^{-1}\right]\right) \\
& \stackrel{n}{\asymp} n^{-\frac{\alpha \min \left(q, p^{\prime}\right)}{2}} \rho\left(n^{\min \left(q, p^{\prime}\right) / 2}\right) .
\end{aligned}
$$

We outline the idea of the proof of Theorems 1 and 2. To obtain the upper estimate, we construct an appropriate partition of the segment, which gives rise to the corresponding space $\mathcal{S}$ of piecewise polynomial functions. Each function $f \in \widehat{W}_{p, g}^{r}\left[0, e^{-1}\right]$ is approximated by an element of the space $\mathcal{S}$; in order to estimate the norm of the difference, we use results of Stepanov, Kufner, and Heinig on the norms of compositions of weighted Riemann-Liouville operators. If $p \geq q$ or $1<p \leq q \leq 2$, then the constructed space of piecewise polynomial functions gives the desired order estimate for Kolmogorov widths. In the case where $q>\max \{p, 2\}$, an analog of Maiorov's discretization method is used: we choose a sequence of partitions and find a subspace of dimension $n$ in the corresponding space $\widetilde{\mathcal{S}}$ of piecewise polynomial functions. For this, we construct an isomorphism between $\widetilde{\mathcal{S}}$ and a space of sequences, and then employ the result of Kashin and Gluskin on widths of finite-dimensional balls.

The lower estimate also can be obtained by constructing an isomorphism between the function space and a space of sequences and by application of well-known results on widths of finite-dimensional balls.

After the proof of Theorem 1, three examples are given, and then Theorem 2 is proved.

In conclusion, we note that in several papers (see [28, 29] and earlier papers) the entropy and the approximation numbers of the embedding operators for weighted Besov and Triebel-Lizorkin classes on $\mathbb{R}^{d}$ were studied. The weights had the form $|x|^{-\beta_{1}}|\ln | x||^{-\gamma_{1}}$ near the origin and $|x|^{-\beta_{2}}|\ln | x||^{-\gamma_{2}}$ near infinity; here $\beta_{1}, \gamma_{1}$ did not affect the asymptotics of the entropy and approximation numbers.

\section{§2. Preliminary Results}

We state Stepanov's criterion (see [22, 23, 24, 25, 26]) for the boundedness of the operator $\widetilde{I}_{r, g, v, b}: L_{p}[a, b) \rightarrow L_{q}[a, b)$ for $1<p, q<+\infty$ (for the operator $I_{r, g, v, a}$, it can be reformulated in a natural way).

Theorem A. Suppose $-\infty<a<b \leq+\infty, r \geq 1$, and $1 \leq q<p \leq \infty$. Then

$$
\left\|\widetilde{I}_{r, g, v, b}\right\|_{L_{p} \rightarrow L_{q}} \underset{p, q, r}{\asymp} A_{0}(p, q, r, g, v, a, b)+A_{1}(p, q, r, g, v, a, b)=: A_{0}+A_{1},
$$

where

$$
\begin{aligned}
& A_{0}=\left(\int_{a}^{b}\left(\int_{a}^{t}(t-x)^{q(r-1)} v^{q}(x) d x\right)^{\frac{p}{p-q}}\left(\int_{t}^{b} g^{p^{\prime}}(y) d y\right)^{\frac{p(q-1)}{p-q}} g^{p^{\prime}}(t) d t\right)^{\frac{1}{q}-\frac{1}{p}} \\
& A_{1}=\left(\int_{a}^{b}\left(\int_{a}^{t} v^{q}(x) d x\right)^{\frac{q}{p-q}}\left(\int_{t}^{b}(y-t)^{p^{\prime}(r-1)} g^{p^{\prime}}(y) d y\right)^{\frac{q(p-1)}{p-q}} v^{q}(t) d t\right)^{\frac{1}{q}-\frac{1}{p}} .
\end{aligned}
$$

Suppose $r \geq 1$ and $1<p \leq q<\infty$. Then

$$
\left\|\widetilde{I}_{r, g, v, b}\right\|_{L_{p} \rightarrow L_{q}} \underset{p, q, r}{\asymp} B_{0}(p, q, r, g, v, a, b)+B_{1}(p, q, r, g, v, a, b)=: B_{0}+B_{1},
$$


where

$$
\begin{aligned}
& B_{0}=\sup _{t \in(a, b)}\left(\int_{a}^{t}(t-x)^{q(r-1)} v^{q}(x) d x\right)^{1 / q}\left(\int_{t}^{b} g^{p^{\prime}}(x) d x\right)^{1 / p^{\prime}}, \\
& B_{1}=\sup _{t \in(a, b)}\left(\int_{a}^{t} v^{q}(x) d x\right)^{1 / q}\left(\int_{t}^{b}(x-t)^{p^{\prime}(r-1)} g^{p^{\prime}}(x) d x\right)^{1 / p^{\prime}} .
\end{aligned}
$$

A criterion for the boundedness of the operators $I_{r, g, v}^{a, b, k}$ and $\widetilde{I}_{r, g, v}^{a, b, k}: L_{p}[a, b] \rightarrow L_{q}[a, b]$ was obtained in the paper [27] by Heinig and Kufner (we formulate this result for $\widetilde{I}_{r, g, v}^{a, b, k}$ ).

Theorem B. Suppose $[a, b] \subset \mathbb{R}, r \in \mathbb{N}$, and $1 \leq k \leq r-1, k \in \mathbb{N}$. If $1 \leq q<p \leq \infty$, then

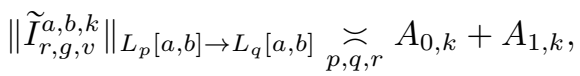

$$
\begin{aligned}
& A_{0, k}^{\frac{p q}{p-q}}=\int_{a}^{b}\left(\int_{t}^{b} v^{q}(x)(x-a)^{q(k-1)} d x\right)^{\frac{p}{p-q}} \\
& \times\left(\int_{a}^{t} g^{p^{\prime}}(x)(x-a)^{p^{\prime}(r-k)} d x\right)^{\frac{p(q-1)}{p-q}}(t-a)^{p^{\prime}(r-k)} g^{p^{\prime}}(t) d t, \\
& A_{1, k}^{\frac{p q}{p-q}}=\int_{a}^{b}\left(\int_{a}^{t} v^{q}(x)(x-a)^{q k} d x\right)^{\frac{p}{p-q}} \\
& \times\left(\int_{t}^{b} g^{p^{\prime}}(x)(x-a)^{p^{\prime}(r-k-1)} d x\right)^{\frac{p(q-1)}{p-q}}(t-a)^{p^{\prime}(r-k-1)} g^{p^{\prime}}(t) d t .
\end{aligned}
$$

If $1<p \leq q<\infty$, then

$$
\begin{gathered}
\left\|\widetilde{I}_{r, g, v}^{a, b, k}\right\|_{L_{p}[a, b] \rightarrow L_{q}[a, b]} \underset{p, \widetilde{q}, r}{\breve{B}} B_{0, k}+B_{1, k}, \\
B_{0, k}=\sup _{t \in(a, b)}\left(\int_{t}^{b} v^{q}(x)(x-a)^{q(k-1)} d x\right)^{1 / q}\left(\int_{a}^{t} g^{p^{\prime}}(x)(x-a)^{p^{\prime}(r-k)} d x\right)^{1 / p^{\prime}}, \\
B_{1, k}=\sup _{t \in(a, b)}\left(\int_{a}^{t} v^{q}(x)(x-a)^{q k} d x\right)^{1 / q}\left(\int_{t}^{b} g^{p^{\prime}}(x)(x-a)^{p^{\prime}(r-k-1)} d x\right)^{1 / p^{\prime}} .
\end{gathered}
$$

For $p \geq q$, Pietsch [7] and Stesin [8] showed that

$$
d_{n}\left(B_{p}^{\nu}, l_{q}^{\nu}\right)=\lambda_{n}\left(B_{p}^{\nu}, l_{q}^{\nu}\right)=(\nu-n)^{\frac{1}{q}-\frac{1}{p}}, \quad n \in \mathbb{Z}_{+}, \quad \nu \in \mathbb{N}, \quad n \leq \nu .
$$

For $p<q$, Kashin [6] and Gluskin [11] obtained the following result.

Theorem C. If $1<p \leq q<\infty$, then

$$
\begin{aligned}
& d_{n}\left(B_{p}^{\nu}, l_{q}^{\nu}\right) \underset{q, p}{\asymp} \Phi(n, \nu, p, q), \\
& \lambda_{n}\left(B_{p}^{\nu}, l_{q}^{\nu}\right) \underset{q, p}{\asymp} \Psi(n, \nu, p, q),
\end{aligned}
$$

where

$$
\begin{aligned}
\Phi(n, \nu, p, q) & = \begin{cases}\min \left\{1,\left(\nu^{1 / q} n^{-1 / 2}\right)\left(\frac{1}{p}-\frac{1}{q}\right) /\left(\frac{1}{2}-\frac{1}{q}\right)\right\} & \text { if } 2 \leq p \leq q<\infty, \\
\max \left\{\nu^{\frac{1}{q}-\frac{1}{p}}, \min \left(1, \nu^{\frac{1}{q}} n^{-\frac{1}{2}}\right)\left(1-\frac{n}{\nu}\right)^{1 / 2}\right\} & \text { if } 1<p<2 \leq q<\infty, \\
\max \left\{\nu^{\frac{1}{q}-\frac{1}{p}}\left(1-\frac{n}{\nu}\right)\left(\frac{1}{q}-\frac{1}{p}\right) /\left(1-\frac{2}{p}\right)\right\} & \text { if } 1<p \leq q \leq 2,\end{cases} \\
\Psi(n, \nu, p, q) & = \begin{cases}\Phi(n, \nu, p, q) & \text { if } q \leq p^{\prime}, \\
\Phi\left(n, \nu, q^{\prime}, p^{\prime}\right) & \text { if } p^{\prime}<q .\end{cases}
\end{aligned}
$$


The widths of Sobolev classes have the following orders:

$$
\begin{aligned}
& d_{n}\left(W_{p}^{r}[0,1], L_{q}[0,1]\right) \underset{p, \widetilde{q}, r}{ } \begin{cases}n^{-r} & \text { if } p \geq q \text { or } 2 \leq p \leq q \leq \infty, \\
n^{-r-\frac{1}{q}+\frac{1}{p}} & \text { if } 1 \leq p \leq q \leq 2, \\
n^{-r-\frac{1}{2}+\frac{1}{p}} & \text { if } 1<p<2 \leq q \leq \infty,\end{cases} \\
& \lambda_{n}\left(W_{p}^{r}[0,1], L_{q}[0,1]\right) \underset{p, \widetilde{q}, r}{ } \begin{cases}n^{-r} & \text { if } p \geq q, \\
n^{-r-\frac{1}{q}+\frac{1}{p}} & \text { if } 1 \leq p \leq q \leq 2 \text { or } 2 \leq p \leq q \leq \infty, \\
n^{-r-\frac{1}{2}+\frac{1}{p}} & \text { if } 1<p<2 \leq q \leq \infty, q \leq p^{\prime}, \\
n^{-r-\frac{1}{q}+\frac{1}{2}} & \text { if } 1 \leq p<2 \leq q<\infty, q>p^{\prime} .\end{cases}
\end{aligned}
$$

\section{§3. Auxiliary assertions}

We formulate some assertions to be proved at the end of this paper.

Suppose $1 \leq q<\infty, 1<p \leq \infty, \frac{1}{s}=\left(\frac{1}{q}-\frac{1}{p}\right)_{+}, r \in \mathbb{N}$, and let $g, v:\left(0, e^{-1}\right] \rightarrow \mathbb{R}_{+}$ have the form

$$
g(x)=x^{-\beta_{g}} \varphi_{g}(|\ln x|), \quad v(x)=x^{-\beta_{v}} \varphi_{v}(|\ln x|),
$$

where $\varphi_{g}, \varphi_{v}:[1,+\infty) \rightarrow(0,+\infty)$ satisfy the conditions of Theorem 1 Let $\beta_{g}+\beta_{v}=$ $r+\frac{1}{q}-\frac{1}{p}, \beta_{v} \neq \frac{1}{q}+k, 0 \leq k \leq r-1$. Throughout, we denote $c_{*}(\varepsilon)=\max \left\{c_{\varphi_{g}}(\varepsilon), c_{\varphi_{v}}(\varepsilon)\right\}$.

Proposition 1. Let $0<a<b \leq e^{-1}, b \leq e a$. Then for any $\sigma, \tau \in[a, b]$ we have

$$
\frac{g(\tau)}{g(\sigma)} \underset{\beta_{g}, c_{*}}{\asymp} 1, \quad \frac{v(\tau)}{v(\sigma)} \underset{\beta_{v}, c_{*}}{\asymp} 1 .
$$

Proposition 2. Let $0<a<b \leq e^{-1}, b \leq e a$, and let $k \in\{0, \ldots, r\}$. Then

$$
\begin{aligned}
\max _{0 \leq k \leq r}\left\|\widetilde{I}_{r, g, v}^{a, b, k}\right\|_{L_{p}[a, b] \rightarrow L_{q}[a, b]} & \underset{p, q, r, \beta_{g}, c_{*}}{\lesssim}\|g v\|_{L_{\varkappa}[a, b]} \\
& \leq\left\|\varphi_{g} \varphi_{v}\right\|_{\left.L_{\infty}[|\ln b|, \mid \ln a]\right]}\left(\ln \frac{b}{a}\right)^{r+\frac{1}{q}-\frac{1}{p}} .
\end{aligned}
$$

Denoting by $\lceil x\rceil$ the integer nearest to $x$ from above, we put

$$
\lceil x\rceil_{0, r}=\max \{0, \min \{r,\lceil x\rceil\}\} .
$$

Proposition 3. Suppose $0<a<b \leq e^{-1}, \beta_{v} \notin\left\{\frac{1}{q}, \frac{1}{q}+1, \ldots, \frac{1}{q}+r-1\right\}, q<p$, and $k=\left\lceil\beta_{v}-\frac{1}{q}\right\rceil_{0, r}$. Then

$$
\left\|\widetilde{I}_{r, g, v}^{a, b, k}\right\|_{L_{p}[a, b] \rightarrow L_{q}[a, b]} \underset{p, q, r, \beta_{g}, c_{*}}{\lesssim}\left\|\varphi_{g} \varphi_{v}\right\|_{L_{\frac{q p}{p-q}}[|\ln b|,|\ln a|]}
$$

Proposition 4. Suppose $0 \leq a<b \leq e^{-1}, 1<p \leq q<\infty, k=\left\lceil\beta_{v}-\frac{1}{q}\right\rceil_{0, r}$, and $\beta_{v} \notin\left\{\frac{1}{q}, \frac{1}{q}+1, \ldots, \frac{1}{q}+r-1\right\}$. Then

$$
\left\|\widetilde{I}_{r, g, v}^{a, b, k}\right\|_{L_{p}[a, b] \rightarrow L_{q}[a, b]} \underset{p, q, r, \beta_{g}, c_{*}}{\lesssim}\left\|\varphi_{g} \varphi_{v}\right\|_{L_{\infty}[|\ln b|,|\ln a|]} \min \left\{1, \ln \frac{b}{a}\right\}^{r+\frac{1}{q}-\frac{1}{p}}
$$

Lemma 1. Suppose $1<p \leq q<\infty, q \geq 2, \omega>\frac{1}{p}, m \in \mathbb{Z}_{+}, n \in \mathbb{N}$, and $\nu(m) \underset{q, p, \omega}{\lesssim} 2^{m} n$. Then there exist sequences $k(m) \in\{0, \ldots, \nu(m)\}$ and $\tilde{k}(m) \in\{0, \ldots, \nu(m)\}$ such that 


$$
\begin{aligned}
\sum_{m \in \mathbb{Z}_{+}} & k(m) \underset{q, \tilde{p}, \omega}{\lesssim} n, \sum_{m \in \mathbb{Z}_{+}} \widetilde{k}(m) \underset{q, p, \omega}{\lesssim} n, \text { and } \\
& \sum_{m=0}^{\infty} d_{k(m)}\left(B_{p}^{\nu(m)}, l_{q}^{\nu(m)}\right)\left(2^{m} n\right)^{-\omega-\frac{1}{q}+\frac{1}{p}} \underset{q, p, \omega}{\lesssim} \begin{cases}n^{-\omega+\frac{1}{p}-\frac{1}{2}} & \text { if } p \leq 2, \\
n^{-\omega} & \text { if } p>2,\end{cases} \\
& \sum_{m=0}^{\infty} \lambda_{\tilde{k}(m)}\left(B_{p}^{\nu(m)}, l_{q}^{\nu(m)}\right)\left(2^{m} n\right)^{-\omega-\frac{1}{q}+\frac{1}{p}} \underset{q, p, \omega}{\lesssim} \begin{cases}n^{-\omega+\frac{1}{p}-\frac{1}{2}} & \text { if } p \leq q^{\prime}, \\
n^{-\omega-\frac{1}{q}+\frac{1}{2}} & \text { if } q^{\prime}<p \leq 2 .\end{cases}
\end{aligned}
$$

This lemma was proved in [20].

Lemma 2. Suppose $\sigma<\tau$ and $\left\{\Delta_{j}\right\}_{j=1}^{j_{0}}$ is a partition of $[\sigma, \tau]$. Also, suppose that $g$, $v:[\sigma, \tau] \rightarrow \mathbb{R}_{+},\left.g\right|_{\Delta_{j}}=c_{j},\left.v\right|_{\Delta_{j}}=a_{j}$, and $1 \leq p, q \leq \infty, r, n, N \in \mathbb{N}, N \geq \max \left\{j_{0}, 2 n\right\}$. Then

$$
\begin{aligned}
& d_{n}\left(\widehat{W}_{p, g}^{r}[\sigma, \tau], L_{q, v}[\sigma, \tau]\right) \underset{p, q, r}{\gtrsim}\|g v\|_{L_{\varkappa}[\sigma, \tau]} N^{-r-\frac{1}{q}+\frac{1}{p}} d_{n}\left(B_{p}^{N}, l_{q}^{N}\right), \\
& \mathcal{A}_{n}\left(\widehat{W}_{p, g}^{r}[\sigma, \tau], L_{q, v}[\sigma, \tau]\right) \underset{p, q, r}{\gtrsim}\|g v\|_{L_{\varkappa}[\sigma, \tau]} N^{-r-\frac{1}{q}+\frac{1}{p}} \lambda_{n}\left(B_{p}^{N}, l_{q}^{N}\right) .
\end{aligned}
$$

Proof. We may assume that $\left\{j=1, \ldots, j_{0}: a_{j} c_{j}>0\right\} \neq \varnothing$. Put $J=\left\{j=1, \ldots, j_{0}\right.$ : $\left.4 N a_{j}^{\varkappa} c_{j}^{\varkappa}\left|\Delta_{j}\right| \geq \sum_{i=1}^{j_{0}} a_{i}^{\varkappa} c_{i}^{\varkappa}\left|\Delta_{i}\right|\right\}, J^{\prime}=\left\{1, \ldots, j_{0}\right\} \backslash J$. Then for any $j \in J^{\prime}$ we have

$$
a_{j}^{\varkappa} c_{j}^{\varkappa}\left|\Delta_{j}\right|-\frac{1}{4 N} \sum_{i \in J^{\prime}} a_{i}^{\varkappa} c_{i}^{\varkappa}\left|\Delta_{i}\right|<\frac{1}{4 N} \sum_{i \in J} a_{i}^{\varkappa} c_{i}^{\varkappa}\left|\Delta_{i}\right| .
$$

Summing over $j \in J^{\prime}$ yields

$$
\sum_{j \in J^{\prime}} a_{j}^{\varkappa} c_{j}^{\varkappa}\left|\Delta_{j}\right|\left(1-\frac{\left|J^{\prime}\right|}{4 N}\right)<\frac{\left|J^{\prime}\right|}{4 N} \sum_{j \in J} a_{j}^{\varkappa} c_{j}^{\varkappa}\left|\Delta_{j}\right| .
$$

Since $\left|J^{\prime}\right| \leq j_{0} \leq N$, we have

$$
\sum_{j \in J^{\prime}} a_{j}^{\varkappa} c_{j}^{\varkappa}\left|\Delta_{j}\right| \leq \frac{1}{3} \sum_{j \in J} a_{j}^{\varkappa} c_{j}^{\varkappa}\left|\Delta_{j}\right| .
$$

For $k \in J$, put

$$
n_{k}=\left[4 N \frac{c_{k}^{\varkappa} a_{k}^{\varkappa}\left|\Delta_{k}\right|}{\sum_{i \in J} c_{i}^{\varkappa} a_{i}^{\varkappa}\left|\Delta_{i}\right|}\right]+1
$$

Then

because

$$
4 N \frac{c_{k}^{\varkappa} a_{k}^{\varkappa}\left|\Delta_{k}\right|}{\sum_{i \in J} c_{i}^{\varkappa} a_{i}^{\varkappa}\left|\Delta_{i}\right|} \leq n_{k} \leq 8 N \frac{c_{k}^{\varkappa} a_{k}^{\varkappa}\left|\Delta_{k}\right|}{\sum_{i \in J} c_{i}^{\varkappa} a_{i}^{\varkappa}\left|\Delta_{i}\right|}
$$

$$
4 N \frac{c_{k}^{\varkappa} a_{k}^{\varkappa}\left|\Delta_{k}\right|}{\sum_{i \in J} c_{i}^{\varkappa} a_{i}^{\varkappa}\left|\Delta_{i}\right|} \geq \frac{\sum_{i=1}^{j_{0}} c_{i}^{\varkappa} a_{i}^{\varkappa}\left|\Delta_{i}\right|}{\sum_{i \in J} c_{i}^{\varkappa} a_{i}^{\varkappa}\left|\Delta_{i}\right|} \geq 1
$$

Let $\varphi \in C_{0}^{\infty}\left(\mathbb{R}, \mathbb{R}_{+}\right)$be a function supported on $(0,1)$,

$$
\int_{0}^{1}\left|\varphi^{(r)}(x)\right|^{p} d x=1, \quad \int_{0}^{1} \varphi(x) d x=\gamma_{r, p}>0 .
$$

For each $i \in J$, we partition $\Delta_{i}$ into $n_{i}$ equal intervals $\Delta_{i, j}=x_{i j}+\frac{\left|\Delta_{i}\right|}{n_{i}}[0,1]$ and set $\varphi_{i j}(x)=\varphi\left(\frac{n_{i}}{\left|\Delta_{i}\right|}\left(x-x_{i j}\right)\right)$,

$$
V=\left\{\sum_{i \in J} \sum_{j=1}^{n_{i}} \xi_{i j} \varphi_{i j}, \xi_{i j} \in \mathbb{R}, i \in J, j=1, \ldots, n_{i}\right\} .
$$


Then

$$
\begin{gathered}
d_{n}\left(\widehat{W}_{p, g}^{r}[\sigma, \tau] \cap V, L_{q, v}[\sigma, \tau]\right) \underset{p, q, r}{\underset{ }{\gtrsim}}\left(\sum_{j \in J} a_{j}^{\varkappa} c_{j}^{\varkappa}\left|\Delta_{j}\right|\right)^{1 / \varkappa} N^{-r-\frac{1}{q}+\frac{1}{p}} d_{n}\left(B_{p}^{N}, l_{q}^{N}\right) \\
\underset{p, q, r}{\gtrsim}\|g v\|_{L_{\varkappa}[\sigma, \tau]} N^{-r-\frac{1}{q}+\frac{1}{p}} d_{n}\left(B_{p}^{N}, l_{q}^{N}\right),
\end{gathered}
$$

and a similar estimate holds true for the approximation numbers. The first inequality in (3.2) can be proved in the same way as in 20.

\section{$\S 4$. Proofs of Main Results}

Proof of Theorem 1. Without loss of generality, we may assume that in (1.6) we have $\alpha<r+\frac{1}{q}-\frac{1}{p}$.

In order to prove the upper estimate in (1.7), it suffices to find a sequence $m_{n} \stackrel{n}{\lesssim} n$ such that

$$
d_{m_{n}}\left(\widehat{W}_{p, g}^{r}\left[0, e^{-1}\right], L_{q, v}\left[0, e^{-1}\right]\right) \stackrel{n}{\lesssim}\left\|\varphi_{g} \varphi_{v}\right\|_{L_{\varkappa}[1, n]} d_{n}\left(W_{p}^{r}[0,1], L_{q}[0,1]\right) .
$$

Indeed, let $c \geq 1$ be such that $m_{n} \leq c n$ for each $n$. To prove the upper estimate, it suffices to consider the case where $m_{n}>n$. Put $k_{n}=\left[\frac{n}{c}\right]$. Then $m_{k_{n}} \leq c k_{n} \leq n$,

$$
\begin{aligned}
d_{n}\left(\widehat{W}_{p, g}^{r}\left[0, e^{-1}\right], L_{q, v}\left[0, e^{-1}\right]\right) & \leq d_{m_{k_{n}}}\left(\widehat{W}_{p, g}^{r}\left[0, e^{-1}\right], L_{q, v}\left[0, e^{-1}\right]\right) \\
& \stackrel{n}{\lesssim}\left\|\varphi_{g} \varphi_{v}\right\|_{L_{\varkappa}\left[1, k_{n}\right]} d_{k_{n}}\left(W_{p}^{r}[0,1], L_{q}[0,1]\right) \\
& \stackrel{n}{\lesssim}\left\|\varphi_{g} \varphi_{v}\right\|_{L_{\varkappa}[1, n]} d_{n}\left(W_{p}^{r}[0,1], L_{q}[0,1]\right) .
\end{aligned}
$$

Indeed, $k_{n} \stackrel{n}{=} n$. Therefore,

$$
d_{k_{n}}\left(W_{p}^{r}[0,1], L_{q}[0,1]\right) \stackrel{n}{=} d_{n}\left(W_{p}^{r}[0,1], L_{q}[0,1]\right) .
$$

Since $k_{n} \leq n$, we have $\left\|\varphi_{g} \varphi_{v}\right\|_{L_{\varkappa}\left[1, k_{n}\right]} \leq\left\|\varphi_{g} \varphi_{v}\right\|_{L_{\varkappa}[1, n]}$.

Let $T=\left\{\Delta_{i}\right\}_{i=1}^{n}$ be a partition. Set

$$
\|f\|_{p, q, T, v}=\left(\sum_{i=1}^{n}\|f\|_{L_{q, v}\left(\Delta_{i}\right)}^{\sigma_{p q}}\right)^{\frac{1}{\sigma_{p q}}}
$$

where $\sigma_{p q}=\min \{q, p\}$, and denote by $L_{p, q, T, v}[a, b]$ the space of measurable functions $f$ such that $\|f\|_{p, q, T, v}<\infty$. Note that $\|f\|_{q, v} \leq\|f\|_{p, q, T, v}$.

For each $k \in\{1, \ldots, n-1\}$, we choose $\widehat{l}_{k} \in \mathbb{N}$ and split $\left[e^{-k-1}, e^{-k}\right]$ into segments $E_{j, k}, 1 \leq j \leq \widehat{l}_{k}$, so as to ensure the following conditions:

$$
\begin{aligned}
\frac{\hat{l}_{k}-1}{n} \int_{e^{-n}}^{e^{-1}} g^{\varkappa}(x) v^{\varkappa}(x) d x & <\int_{e^{-k-1}}^{e^{-k}} g^{\varkappa}(x) v^{\varkappa}(x) d x \leq \frac{\widehat{l}_{k}}{n} \int_{e^{-n}}^{e^{-1}} g^{\varkappa}(x) v^{\varkappa}(x) d x, \\
\int_{E_{j, k}} g^{\varkappa}(x) v^{\varkappa}(x) d x & =\frac{1}{\widehat{l}_{k}} \int_{e^{-k-1}}^{e^{-k}} g^{\varkappa}(x) v^{\varkappa}(x) d x \leq \frac{1}{n} \int_{e^{-n}}^{e^{-1}} g^{\varkappa}(x) v^{\varkappa}(x) d x
\end{aligned}
$$

(by condition (\#), the functions $g$ and $v$ are positive everywhere; hence, the $\widehat{l}_{k}$ and $E_{j, k}$ are well defined).

Denote $T_{0,0}=\left\{E_{j, k}: 1 \leq k \leq n-1,1 \leq j \leq \hat{l}_{k}\right\}=\left\{\Delta_{i}^{0}\right\}_{i=1}^{\widehat{n}}$, where

$$
\widehat{n}=\left|T_{0,0}\right|=\sum_{k=1}^{n-1} \widehat{l}_{k} \leq n+\sum_{k=1}^{n-1}\left(\widehat{l}_{k}-1\right) \leq n+\sum_{k=1}^{n-1} n \frac{\|g v\|_{L_{\varkappa}\left[e^{-k-1}, e^{-k}\right]}^{\varkappa}}{\|g v\|_{L_{\varkappa}\left[e^{-n}, e^{-1}\right]}^{\varkappa}} \leq 2 n .
$$


For $m \in \mathbb{N}$, suppose that the partition $T_{0, m-1}=\left\{\Delta_{i}^{m-1}\right\}_{i=1}^{2^{m-1} \hat{n}}$ has been constructed. We construct the partition $T_{0, m}=\left\{\Delta_{i}^{m}\right\}_{i=1}^{2^{m} \hat{n}}=\left\{\left[a_{i}^{m}, b_{i}^{m}\right]\right\}_{i=1}^{2^{m} \hat{n}}$ by dividing each segment $\Delta_{i}^{m-1}$ into two subsegments $\Delta^{\prime}$ and $\Delta^{\prime \prime}$ such that

$$
\int_{\Delta^{\prime}} g^{\varkappa}(x) v^{\varkappa}(x) d x=\int_{\Delta^{\prime \prime}} g^{\varkappa}(x) v^{\varkappa}(x) d x=\frac{1}{2} \int_{\Delta_{i}^{m-1}} g^{\varkappa}(x) v^{\varkappa}(x) d x .
$$

Then

$$
\begin{aligned}
\left|T_{0, m}\right| & \leq 2^{m+1} n \\
\int_{\Delta_{i}^{m}} g^{\varkappa}(x) v^{\varkappa}(x) d x & \leq \frac{1}{2^{m} n} \int_{e^{-n}}^{e^{-1}} g^{\varkappa}(x) v^{\varkappa}(x) d x .
\end{aligned}
$$

Let $f \in \widehat{W}_{p, g}^{r}\left[0, e^{-1}\right]$, let $P_{m} f$ be a piecewise polynomial function defined on $\left[e^{-n}, e^{-1}\right]$, and let $\left.\left(P_{m} f\right)\right|_{\Delta_{i}^{m}}$ be the Taylor polynomial for $f$ at the point $a_{i}^{m}$. We claim that

$$
\begin{gathered}
\left\|f-P_{m} f\right\|_{p, q, T_{0, m}, v} \underset{p, q, r, \beta_{g}, c_{*}}{\lesssim}\left(2^{m} n\right)^{\left(\frac{1}{p}-\frac{1}{q}\right)_{+}{ }^{-r}}\|g v\|_{L_{\varkappa}\left[e^{-n}, e^{-1}\right]} \\
=\left(2^{m} n\right)^{\left(\frac{1}{p}-\frac{1}{q}\right)_{+}-r}\left\|\varphi_{g} \varphi_{v}\right\|_{L_{\varkappa}[1, n]} .
\end{gathered}
$$

Indeed, from Proposition 2 it follows that

$$
\left\|f-P_{m} f\right\|_{L_{q, v}\left(\Delta_{i}^{m}\right)} \underset{p, q, r, \beta_{g}, c_{*}}{\lesssim}\|g v\|_{L_{\varkappa}\left(\Delta_{i}^{m}\right)}\left\|\frac{f^{(r)}}{g}\right\|_{L_{p}\left(\Delta_{i}^{m}\right)} .
$$

Therefore,

$$
\left\|f-P_{m} f\right\|_{p, q, T_{0, m}, v} \underset{p, q, r, \beta_{g}, c_{*}}{\lesssim}\left(\sum_{i=1}^{2^{m} \hat{n}}\|g v\|_{L_{\varkappa}\left(\Delta_{i}^{m}\right)}^{\sigma_{p q}}\left\|\frac{f^{(r)}}{g}\right\|_{L_{p}\left(\Delta_{i}^{m}\right)}^{\sigma_{p q}}\right)^{\frac{1}{\sigma_{p q}}}=: S .
$$

If $p>q$, then

$$
\begin{aligned}
S & \stackrel{4.2}{\leq}\left(\sum_{i=1}^{2^{m} \hat{n}} \frac{1}{\left(2^{m} n\right)^{q / \varkappa}}\|g v\|_{L_{\varkappa}\left[e^{-n}, e^{-1}\right]}^{q}\left\|\frac{f^{(r)}}{g}\right\|_{L_{p}\left(\Delta_{i}^{m}\right)}^{q}\right)^{\frac{1}{q}} \\
& =\frac{1}{\left(2^{m} n\right)^{1 / \varkappa}}\|g v\|_{L_{\varkappa}\left[e^{-n}, e^{-1}\right]}\left(\sum_{i=1}^{2^{m} \hat{n}}\left\|\frac{f^{(r)}}{g}\right\|_{L_{p}\left(\Delta_{i}^{m}\right)}^{q}\right)^{\frac{1}{q}} \\
& \stackrel{{ }_{p, q}^{4.1}}{\lesssim} \frac{1}{\left(2^{m} n\right)^{1 / \varkappa}}\|g v\|_{L_{\varkappa}\left[e^{-n}, e^{-1}\right]}\left(2^{m} n\right)^{\frac{1}{q}-\frac{1}{p}}\left(\sum_{i=1}^{2^{m} \hat{n}}\left\|\frac{f^{(r)}}{g}\right\|_{L_{p}\left(\Delta_{i}^{m}\right)}^{p}\right)^{\frac{1}{p}} \\
& \leq \frac{1}{\left(2^{m} n\right)^{r}}\|g v\|_{L_{\varkappa}\left[e^{-n}, e^{-1}\right]} .
\end{aligned}
$$

If $p \leq q$, then

$$
\begin{aligned}
S & \stackrel{\sqrt[4.2]{\leq}}{\leq}\left(\sum_{i=1}^{2^{m} \hat{n}} \frac{1}{\left(2^{m} n\right)^{p / \varkappa}}\|g v\|_{L_{\varkappa}\left[e^{-n}, e^{-1}\right]}^{p}\left\|\frac{f^{(r)}}{g}\right\|_{L_{p}\left(\Delta_{i}^{m}\right)}^{p}\right)^{\frac{1}{p}} \\
& =\frac{1}{\left(2^{m} n\right)^{1 / \varkappa}}\|g v\|_{L_{\varkappa}\left[e^{-n}, e^{-1}\right]}\left(\sum_{i=1}^{2^{m} \hat{n}}\left\|\frac{f^{(r)}}{g}\right\|_{L_{p}\left(\Delta_{i}^{m}\right)}^{p}\right)^{\frac{1}{p}} \\
& \leq\|g v\|_{L_{\varkappa}\left[e^{-n}, e^{-1}\right]} \frac{1}{\left(2^{m} n\right)^{r+\frac{1}{q}-\frac{1}{p}}} .
\end{aligned}
$$


Set $j_{*}=\left\lceil\beta_{v}-\frac{1}{q}\right\rceil_{0, r}$. For $0 \leq \sigma<\tau \leq e^{-1}$, we denote by $P^{\sigma, \tau} f$ the polynomial of degree at most $r-1$ such that $f-P^{\sigma, \tau} f=\frac{1}{\left(j_{*}-1\right) !\left(r-j_{*}-1\right) !} \widetilde{I}_{r, 1,1}^{\sigma, \tau, j_{*}} f^{(r)}$. Then we can use (1.5) and Propositions 3 and 4 to get

$$
\left\|f-P^{0, e^{-n}} f\right\|_{L_{q, v}\left[0, e^{-n}\right]} \stackrel{n}{\lesssim}\left\|\varphi_{g} \varphi_{v}\right\|_{L_{\frac{q p}{p-q}}[n, \infty)} \stackrel{n}{\lesssim} n^{-r}\left\|\varphi_{g} \varphi_{v}\right\|_{L_{\varkappa}[1, n]}
$$

for $q<p$ and

$$
\left\|f-P^{0, e^{-n}} f\right\|_{L_{q, v}\left[0, e^{-n}\right]} \stackrel{n}{\lesssim}\left\|\varphi_{g} \varphi_{v}\right\|_{L_{\infty}[n, \infty)} \stackrel{n}{\lesssim} n^{-r-\frac{1}{q}+\frac{1}{p}}\left\|\varphi_{g} \varphi_{v}\right\|_{L_{\varkappa}[1, n]}
$$

for $q \geq p$. Put $\left.(P f)\right|_{\left[e^{-n}, e^{-1}\right]}=P_{0} f,\left.(P f)\right|_{\left[0, e^{-n}\right]}=\left.\left(P^{0, e^{-n}} f\right)\right|_{\left[0, e^{-n}\right]}$. Then relations (4.3), (4.4), and (4.5) imply that

$$
\|f-P f\|_{L_{q, v}\left[0, e^{-1}\right]} \stackrel{n}{\lesssim} n^{\left(\frac{1}{p}-\frac{1}{q}\right)_{+}{ }^{-r}}\left\|\varphi_{g} \varphi_{v}\right\|_{L_{\varkappa}[1, n]} .
$$

Hence, the upper estimate (1.7) is proved for $q \leq p$ and for $p \leq q \leq 2$.

Let $p<q$ and $q>2$. By Proposition 4 and (1.6), for any $k \in \mathbb{N}$ we have

$$
\left\|f-P^{0, e^{-2^{k} \cdot n}} f\right\|_{L_{q, v}\left[0, e^{-2^{k}}\right]} \underset{p, q, r, \beta_{g}, c_{*}}{\lesssim}\left\|\varphi_{g} \varphi_{v}\right\|_{L_{\infty}\left[2^{k} n, \infty\right)} \leq M \cdot 2^{-k \alpha}\left\|\varphi_{g} \varphi_{v}\right\|_{L_{\infty}[n, \infty)} .
$$

Set $k_{0}=\left[\frac{1}{\alpha}\left(\min \left\{\frac{1}{2}, \frac{1}{p}\right\}-\frac{1}{q}\right) \log _{2} n\right]$. Then

$$
\left\|f-P^{0, e^{-2^{k_{0} \cdot n}}} f\right\|_{L_{q, v}\left[0, e^{\left.-2^{k_{0}}\right]}\right]} \underset{p, q, r, \widetilde{\beta_{g}, c_{*}, M}}{\lesssim} n^{-\min \left\{\frac{1}{2}, \frac{1}{p}\right\}+\frac{1}{q}}\left\|\varphi_{g} \varphi_{v}\right\|_{L_{\infty}[n, \infty)} .
$$

We pick a number $\gamma>2$ (to be chosen later). For any $m \in \mathbb{Z}_{+}$and $k=0, \ldots, k_{0}+1$, we define a piecewise polynomial function $P_{k, m} f$ as follows. Set

$$
\begin{aligned}
\left(P_{0, m} f\right)(x) & =P_{m} f(x) \text { for } x \in\left[e^{-n}, e^{-1}\right], \quad\left(P_{0, m} f\right)(x)=0 \text { for } x \in\left[0, e^{-n}\right) ; \\
\left(P_{k_{0}+1,0} f\right)(x) & =P^{0, e^{-2^{k_{0}} n}}(x) \text { for } x \in\left[0, e^{-2^{k_{0}} n}\right], \\
\left(P_{k_{0}+1,0} f\right)(x) & =0 \text { for } x \notin\left[0, e^{-2^{k_{0}} n}\right] .
\end{aligned}
$$

For $1 \leq k \leq k_{0}$, consider the partition $T_{k, m}=\left\{\Delta_{k, m, j}\right\}_{j=1}^{N_{k, m}}$ of $\left[e^{-2^{k} n}, e^{-2^{k-1} n}\right]$ by the points $e^{-2^{-m} \gamma^{k} j}$; here $j \in \mathbb{N}$ and $2^{k-1} n \leq 2^{-m} \gamma^{k} j \leq 2^{k} n$. If $\gamma$ is sufficiently close to 2 , then for any $k \in\left\{1, \ldots, k_{0}\right\}$ and $m \in \mathbb{Z}_{+}$we have $\left(\frac{2}{\gamma}\right)^{k} 2^{m} n \geq 1$. Therefore,

$$
N_{k, m} \stackrel{n, m, k, \gamma}{=}\left(\frac{2}{\gamma}\right)^{k} 2^{m} n \text {. }
$$

If $[\sigma, \tau] \in T_{k, m}$, then

$$
\ln \frac{\tau}{\sigma} \leq 2^{-m} \gamma^{k} .
$$

If $m \geq 1$, then the partition $T_{k, m}$ is a subpartition of $T_{k, m-1}$, and each element of $T_{k, m-1}$ contains at most two elements of $T_{k, m}$. For $x \notin\left[e^{-2^{k} n}, e^{-2^{k-1} n}\right]$ we put $P_{k, m} f=0$; if $[\sigma, \tau] \in T_{k, m}$, then we set $\left.\left(P_{k, m} f\right)\right|_{(\sigma, \tau)}=\left.\left(P^{\sigma, \tau} f\right)\right|_{(\sigma, \tau)}$. Since $f \in \widehat{W}_{p, g}^{r}\left[0, e^{-1}\right]$, it follows 
that

$$
\begin{aligned}
& \left\|P_{0, m} f-P_{0, m-1} f\right\|_{p, q, T_{0, m}, v} \underset{p, q, r, \beta_{g}, c_{*}}{\stackrel{4.3}{\lesssim}}\left(2^{m} n\right)^{-r-\frac{1}{q}+\frac{1}{p}}\left\|\varphi_{g} \varphi_{v}\right\|_{L_{\varkappa}[1, n]}, \\
& \left\|P_{k, m} f-P_{k, m-1} f\right\|_{p, q, T_{k, m}, v} \\
& \underset{p, q}{\lesssim}\left(\sum_{[\sigma, \tau] \in T_{k, m}}\left\|\widetilde{I}_{r, g, v}^{\sigma, \tau, j_{*}}\right\|_{L_{p}[\sigma, \tau] \rightarrow L_{q}[\sigma, \tau]}^{p}\left\|\frac{f^{(r)}}{g}\right\|_{L_{p}[\sigma, \tau]}^{p}\right)^{\frac{1}{p}} \\
& +\left(\sum_{[\sigma, \tau] \in T_{k, m-1}}\left\|\widetilde{I}_{r, g, v}^{\sigma, \tau, j_{*}}\right\|_{L_{p}[\sigma, \tau] \rightarrow L_{q}[\sigma, \tau]}^{p}\left\|\frac{f^{(r)}}{g}\right\|_{L_{p}[\sigma, \tau]}^{p}\right)^{\frac{1}{p}} \\
& \leq \max _{[\sigma, \tau] \in T_{k, m}}\left\|\widetilde{I}_{r, g, v}^{\sigma, \tau, j_{*}}\right\|_{L_{p}[\sigma, \tau] \rightarrow L_{q}[\sigma, \tau]}+\max _{[\sigma, \tau] \in T_{k, m-1}}\left\|\widetilde{I}_{r, g, v}^{\sigma, \tau, j_{*}}\right\|_{L_{p}[\sigma, \tau] \rightarrow L_{q}[\sigma, \tau]}
\end{aligned}
$$

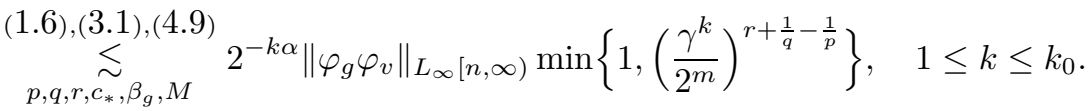

Hence,

$$
f=\sum_{0 \leq k \leq k_{0}+1} P_{k, 0} f+\sum_{m \in \mathbb{N}} \sum_{0 \leq k \leq k_{0}}\left(P_{k, m} f-P_{k, m-1} f\right)+\left(f \cdot \chi_{\left[0, e^{\left.-2^{k_{0}}\right]}\right.}-P_{k_{0}+1,0} f\right)
$$

(the sum is taken in $L_{q, v}$ ).

Suppose $0 \leq k \leq k_{0}, m \in \mathbb{N}$, and $\Delta \in T_{k, m}$. Let $\mathcal{P}^{r-1}(\Delta)$ be the space of polynomials of degree at most $r-1$ defined on the segment $\Delta$, and let $A_{k, m, \Delta}: \mathcal{P}^{r-1}(\Delta) \rightarrow l_{2}^{r}$

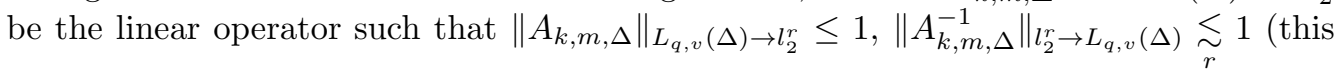
operator exists by John's theorem on ellipsoids, see, e.g., [30]). Denote by $\mathcal{S}_{r, T_{k, m}}$ the set of functions whose restriction on each interval $\Delta \in T_{k, m}$ is a polynomial of degree at most $r-1, \nu_{k, m}=\operatorname{dim} \mathcal{S}_{r, T_{k, m}} \underset{\widehat{r}}{\stackrel{4.8}{\widetilde{r}}}\left(\frac{2}{\gamma}\right)^{k} 2^{m} n$. For any $S \in \mathcal{S}_{r, T_{k, m}}$ we set $A_{k, m}(S)=$ $\left\{A_{k, m, \Delta}\left(\left.S\right|_{\Delta}\right)\right\}_{\Delta \in T_{k, m}}$. Then

$$
\begin{aligned}
&\left\|A_{k, m} S\right\|_{l_{p}^{\nu_{k, m}}}=\left(\sum_{\Delta \in T_{k, m}}\left\|A_{k, m, \Delta}\left(\left.S\right|_{\Delta}\right)\right\|_{l_{p}^{r}}^{p}\right)^{\frac{1}{p}} \\
& \underset{p, r}{\lesssim}\left(\sum_{\Delta \in T_{k, m}}\|S\|_{L_{q, v}}^{p}(\Delta)\right)^{\frac{1}{p}}=\|S\|_{p, q, T_{k, m}, v}, \\
&\left\|A_{k, m}^{-1}\left(c_{\Delta, j}\right)_{\Delta \in T_{k, m}, 1 \leq j \leq r}\right\|_{L_{q, v}}=\left(\sum_{\Delta \in T_{k, m}}\left\|A_{k, m, \Delta}^{-1}\left(c_{\Delta, j}\right)_{1 \leq j \leq r}\right\|_{L_{q, v}(\Delta)}^{q}\right)^{\frac{1}{q}} \\
& \underset{q, r}{\lesssim}\left(\sum_{\Delta \in T_{k, m}}\left\|\left(c_{\Delta, j}\right)_{1 \leq j \leq r}\right\|_{l_{q}^{r}}^{q}\right)^{\frac{1}{q}}=\left\|\left(c_{\Delta, j}\right)_{\Delta \in T_{k, m}, 1 \leq j \leq r}\right\|_{l_{q}^{\nu_{k, m}}} .
\end{aligned}
$$

Suppose $l_{k, m} \in \mathbb{Z}_{+}, k, m \in \mathbb{N}, k \leq k_{0}+1$, and $\sum_{k, m} l_{k, m} \leq C n$ for some $C>0$. Let $\Lambda_{k, m} \subset l_{q}^{\nu_{k, m}}$ be the extremal subspace for $d_{l_{k, m}}\left(B_{p}^{\nu_{k, m}}, l_{q}^{\nu_{k, m}}\right)$, let $I_{k, m}: l_{q}^{\nu_{k, m}} \rightarrow l_{q}^{\nu_{k, m}}$ be the identity operator, and let $E_{k, m}: l_{q}^{\nu_{k, m}} \rightarrow \Lambda_{k, m}$ be the metric projection onto $\Lambda_{k, m}$. For each function $f \in \widehat{W}_{p, g}^{r}\left[0, e^{-1}\right]$, we set

$$
P f=\sum_{0 \leq k \leq k_{0}+1} P_{k, 0} f+\sum_{m \in \mathbb{N}} \sum_{0 \leq k \leq k_{0}} A_{k, m}^{-1} E_{k, m} A_{k, m}\left(P_{k, m} f-P_{k, m-1} f\right) .
$$


Then the image of the map $P$ is contained in some subspace $V \subset L_{q, v}\left[0, e^{-1}\right]$ (since $f$ and $\widetilde{I}_{r, 1,1}^{0, \tau, j_{*}} f^{(r)}$ belong to $L_{q, v}$ for any $\tau \in\left[0, e^{-1}\right]$, we have $\left.P_{k_{0}+1,0} f \in L_{q, v}\right)$; here

$$
\operatorname{dim} V \leq r \widehat{n}+r \sum_{k=1}^{k_{0}}\left(n\left(\frac{2}{\gamma}\right)^{k}+1\right)+r+\sum_{m \in \mathbb{N}} \sum_{k=0}^{k_{0}} l_{k, m} \stackrel{n}{\lesssim} n
$$

and

$$
\begin{aligned}
& \|f-P f\|_{L_{q, v}\left[0, e^{-1}\right]} \stackrel{(4.12)}{\leq}\left\|f-P_{k_{0}+1,0} f\right\|_{L_{q, v}\left[0, e^{-2^{k_{0}}}\right]} \\
& +\sum_{m \in \mathbb{N}} \sum_{0 \leq k \leq k_{0}}\left\|A_{m, k}^{-1}\left(I_{k, m}-E_{k, m}\right) A_{k, m}\left(P_{k, m} f-P_{k, m-1} f\right)\right\|_{L_{q, v}\left[0, e^{-1}\right]} \\
& \text { 4.7, 4.13, 4.14 } \\
& \underset{p, q, r, r, \beta_{g}, c_{*}}{\lesssim} n^{-\min \left\{\frac{1}{2}, \frac{1}{p}\right\}+\frac{1}{q}\left\|\varphi_{g} \varphi_{v}\right\|_{L_{\infty}[n, \infty)}} \\
& +\sum_{m \in \mathbb{N}} \sum_{0 \leq k \leq k_{0}} d_{l_{k, m}}\left(B_{p}^{\nu_{k, m}}, l_{q}^{\nu_{k, m}}\right)\left\|P_{k, m} f-P_{k, m-1} f\right\|_{p, q, T_{k, m}, v} \\
& \underset{p, q, r, M_{,}, \beta_{g}, c_{*}}{\stackrel{4.10}{\text {,4.11 }}} n^{-\min \left\{\frac{1}{2}, \frac{1}{p}\right\}+\frac{1}{q}}\left\|\varphi_{g} \varphi_{v}\right\|_{L_{\infty}[n, \infty)} \\
& +\sum_{m \in \mathbb{N}} d_{l_{0, m}}\left(B_{p}^{\nu_{0, m}}, l_{q}^{\nu_{0, m}}\right)\left(2^{m} n\right)^{-r-\frac{1}{q}+\frac{1}{p}}\left\|\varphi_{g} \varphi_{v}\right\|_{L_{\varkappa}[1, n]} \\
& +\sum_{m \in \mathbb{N}} \sum_{1 \leq k \leq k_{0}} d_{l_{k, m}}\left(B_{p}^{\nu_{k, m}}, l_{q}^{\nu_{k, m}}\right) 2^{-k \alpha}\left\|\varphi_{g} \varphi_{v}\right\|_{L_{\infty}[n, \infty)} \min \left\{1,\left(\frac{\gamma^{k}}{2^{m}}\right)^{r+\frac{1}{q}-\frac{1}{p}}\right\} .
\end{aligned}
$$

By Lemma 1, there exists a sequence $l_{0, m}$ such that $\sum_{m} l_{0, m} \underset{p, q, r}{\lesssim} n$ and

$$
\sum_{m \in \mathbb{N}} d_{l_{0, m}}\left(B_{p}^{\nu_{0, m}}, l_{q}^{\nu_{0, m}}\right)\left(2^{m} n\right)^{-r-\frac{1}{q}+\frac{1}{p}}\left\|\varphi_{g} \varphi_{v}\right\|_{L_{\varkappa}[1, n]} \underset{p, q, r}{\lesssim}\left\|\varphi_{g} \varphi_{v}\right\|_{L_{\varkappa}[1, n]} n^{-r+\left(\frac{1}{p}-\frac{1}{2}\right)_{+}}
$$

Condition (1.5) shows that $\left\|\varphi_{g} \varphi_{v}\right\|_{L_{\infty}[n, \infty)} \stackrel{n}{\lesssim}\left\|\varphi_{g} \varphi_{v}\right\|_{L_{\varkappa}[1, n]} n^{-r-\frac{1}{q}+\frac{1}{p}}$. Therefore, it remains to find a sequence $\left(l_{k, m}\right)=\left(l_{k, m}^{(n)}\right)$ such that

$$
\sum_{1 \leq k \leq k_{0}} \sum_{m \in \mathbb{N}} l_{k, m}^{(n)} \stackrel{n}{\lesssim} n
$$

and

$$
\sum_{m \in \mathbb{N}} \sum_{1 \leq k \leq k_{0}} d_{l_{k, m}^{(n)}}\left(B_{p}^{\nu_{k, m}}, l_{q}^{\nu_{k, m}}\right) 2^{-k \alpha} \min \left\{1,\left(\frac{\gamma^{k}}{2^{m}}\right)^{r+\frac{1}{q}-\frac{1}{p}}\right\} \sum^{n} n^{\frac{1}{q}-\min \left\{\frac{1}{2}, \frac{1}{p}\right\}} .
$$

Let $\tau<1$ (this number will be chosen later). Set $m_{0}=\left[c \log _{2} n\right]$, where

$$
c=\frac{\min \left\{\frac{1}{2}, \frac{1}{p}\right\}-\frac{1}{q}}{\alpha \log _{\gamma} 2}, \quad l_{k, m}=0 \text { if } m>m_{0}, \quad l_{k, m}=\left[\tau^{m}\left(\frac{2}{\gamma}\right)^{k} n\right] \quad \text { if } m \leq m_{0} .
$$

Since $\tau<1$ and $\gamma>2$, we have $\sum_{k, m} l_{k, m} \underset{\tau, \gamma}{\lesssim} n$. 
The sum (4.16) over $m>m_{0}$ is equal to

$$
\begin{aligned}
\sum_{m>\left[c \log _{2} n\right]} & \sum_{1 \leq k \leq k_{0}} 2^{-k \alpha} \min \left\{1,\left(\frac{\gamma^{k}}{2^{m}}\right)^{r+\frac{1}{q}-\frac{1}{p}}\right\} \\
= & \sum_{m>\left[c \log _{2} n\right]} \sum_{k \geq m \frac{\ln 2}{\ln \gamma}} 2^{-k \alpha}+\sum_{m>\left[c \log _{2}\right.} \sum_{n]<m \frac{\ln 2}{\ln \gamma}} 2^{-k \alpha}\left(\frac{\gamma^{k}}{2^{m}}\right)^{r+\frac{1}{q}-\frac{1}{p}} .
\end{aligned}
$$

The first sum equals

$$
\begin{aligned}
\sum_{k=1}^{k_{0}} \sum_{\left[c \log _{2}\right.} \sum_{n]<m \leq k \frac{\ln \gamma}{\ln 2}} 2^{-k \alpha} \leq \sum_{k \geq \frac{\ln 2}{\ln \gamma}\left[c \log _{2} n\right]} 2^{-k \alpha}\left(k \frac{\ln \gamma}{\ln 2}-\left[c \log _{2} n\right]\right) \\
\asymp 2^{-\left(\alpha c \log _{\gamma} 2\right) \log _{2} n}=n^{-\alpha c \log _{\gamma} 2}=n^{\frac{1}{q}-\min \left\{\frac{1}{2}, \frac{1}{p}\right\}} .
\end{aligned}
$$

Since $\alpha<r+\frac{1}{q}-\frac{1}{p}$, the order of the second sum does not exceed

$$
\begin{aligned}
& \sum_{m>\left[c \log _{2} n\right]} 2^{-\left(r+\frac{1}{q}-\frac{1}{p}\right) m} \gamma^{\left(r+\frac{1}{q}-\frac{1}{p}\right) m \log _{\gamma} 2} 2^{-\alpha m \log _{\gamma} 2} \\
& \quad=\sum_{m>\left[c \log _{2} n\right]} 2^{-\alpha m \log _{\gamma} 2} \lesssim 2^{-\left(\alpha c \log _{\gamma} 2\right) \log _{2} n}=n^{-\alpha c \log _{\gamma} 2}=n^{\frac{1}{q}-\min \left\{\frac{1}{2}, \frac{1}{p}\right\}} .
\end{aligned}
$$

Using (2.2) and the order relation $\nu_{k, m} \underset{r}{\asymp}\left[2^{m}\left(\frac{2}{\gamma}\right)^{k} n\right]=: \widetilde{\nu}_{k, m}$, we see that if $p<2$ and $m \leq m_{0}$, then

$$
\begin{aligned}
& d_{l_{k, m}}\left(B_{p}^{\nu_{k, m}}, l_{q}^{\nu_{k, m}}\right) \underset{p, q, r}{\lesssim} d_{l_{k, m}}\left(B_{p}^{\widetilde{\nu}_{k, m}}, l_{q}^{\widetilde{\nu}_{k, m}}\right) \\
& \quad \underset{p, q}{\lesssim} 2^{\frac{m}{q}}\left(\frac{2}{\gamma}\right)^{\frac{k}{q}} n^{\frac{1}{q}} \tau^{-\frac{m}{2}}\left(\frac{2}{\gamma}\right)^{-\frac{k}{2}} n^{-\frac{1}{2}}=n^{\frac{1}{q}-\frac{1}{2}}\left(\frac{2}{\gamma}\right)^{k\left(\frac{1}{q}-\frac{1}{2}\right)} 2^{\frac{m}{q}} \tau^{-\frac{m}{2}},
\end{aligned}
$$

and if $p \geq 2$ and $m \leq m_{0}$, then

$$
\begin{aligned}
d_{l_{k, m}}\left(B_{p}^{\nu_{k, m}}, l_{q}^{\nu_{k, m}}\right) \underset{p, q, r}{\lesssim} d_{l_{k, m}}\left(B_{p}^{\widetilde{\nu}_{k, m}}, l_{q}^{\widetilde{\nu}_{k, m}}\right) \\
\quad \underset{p, q}{\lesssim}\left(n^{\frac{1}{q}-\frac{1}{2}}\left(\frac{2}{\gamma}\right)^{k\left(\frac{1}{q}-\frac{1}{2}\right)} 2^{\frac{m}{q}} \tau^{-\frac{m}{2}}\right)^{\left(\frac{1}{p}-\frac{1}{q}\right) /\left(\frac{1}{2}-\frac{1}{q}\right)} \\
=n^{\frac{1}{q}-\frac{1}{p}}\left(\frac{2}{\gamma}\right)^{k\left(\frac{1}{q}-\frac{1}{p}\right)} 2^{\frac{m}{q} \lambda} \tau^{-\frac{m}{2} \lambda}
\end{aligned}
$$

where $\lambda=\frac{\frac{1}{p}-\frac{1}{q}}{\frac{1}{2}-\frac{1}{q}}$. We estimate the sum of (4.16) over $m \leq m_{0}$ for $\gamma=\gamma(\alpha, p, q)$ sufficiently close to 2 and $\tau=\tau(\alpha, p, q)$ sufficiently close to 1 .

- for $p<2$, we estimate the sum over $m \leq k \frac{\ln \gamma}{\ln 2}$ :

$$
\begin{aligned}
& \sum_{k} \sum_{m \leq k \frac{\ln \gamma}{\ln 2}} d_{l_{k, m}}\left(B_{p}^{\nu_{k, m}}, l_{q}^{\nu_{k, m}}\right) 2^{-k \alpha} \underset{p, q, r}{\stackrel{4.17}{\lesssim}} \sum_{k} \sum_{m \leq k \frac{\ln \gamma}{\ln 2}} 2^{-k \alpha} n^{\frac{1}{q}-\frac{1}{2}}\left(\frac{2}{\gamma}\right)^{k\left(\frac{1}{q}-\frac{1}{2}\right)} 2^{\frac{m}{q}} \tau^{-\frac{m}{2}} \\
& \underset{q, \tau}{\asymp} n^{\frac{1}{q}-\frac{1}{2}} \sum_{k}\left(\frac{2}{\gamma}\right)^{k\left(\frac{1}{q}-\frac{1}{2}\right)} 2^{-k \alpha}\left(2^{\frac{1}{q}} \tau^{-\frac{1}{2}}\right)^{k \frac{\ln \gamma}{\ln 2}} \\
&=n^{\frac{1}{q}-\frac{1}{2}} \sum_{k} 2^{-k\left(\alpha-\frac{1}{q} \frac{\ln \gamma}{\ln 2}\right)} \tau^{-\frac{1}{2} k \frac{\ln \gamma}{\ln 2}}\left(\frac{2}{\gamma}\right)^{k\left(\frac{1}{q}-\frac{1}{2}\right)} \lesssim n^{\frac{n}{q}-\frac{1}{2}}
\end{aligned}
$$

(because $\alpha>\frac{1}{q}$ ). 
- for $p<2$, we estimate the sum over $m>k \frac{\ln \gamma}{\ln 2}$ :

$$
\begin{aligned}
& \sum_{m} \sum_{k<m \frac{\ln 2}{\ln \gamma}} d_{l_{k, m}}\left(B_{p}^{\nu_{k, m}}, l_{q}^{\nu_{k, m}}\right) 2^{-k \alpha}\left(\frac{\gamma^{k}}{2^{m}}\right)^{r+\frac{1}{q}-\frac{1}{p}} \\
& \underset{p, q, r}{\stackrel{4.17}{\lesssim}} \sum_{m} \sum_{k<m \frac{\ln 2}{\ln \gamma}} 2^{-k \alpha} n^{\frac{1}{q}-\frac{1}{2}}\left(\frac{2}{\gamma}\right)^{k\left(\frac{1}{q}-\frac{1}{2}\right)} 2^{\frac{m}{q}} \tau^{-\frac{m}{2}}\left(\frac{\gamma^{k}}{2^{m}}\right)^{r+\frac{1}{q}-\frac{1}{p}} \\
& =n^{\frac{1}{q}-\frac{1}{2}} \sum_{m} \sum_{k<m \frac{\ln 2}{\ln \gamma}} 2^{-k \alpha} \gamma^{k\left(r+\frac{1}{q}-\frac{1}{p}\right)}\left(\frac{2}{\gamma}\right)^{k\left(\frac{1}{q}-\frac{1}{2}\right)} 2^{m\left(-r+\frac{1}{p}\right)} \tau^{-\frac{m}{2}} \\
& \underset{p, q, r, \alpha, \gamma}{\lesssim} n^{\frac{1}{q}-\frac{1}{2}} \sum_{m} 2^{m\left(-r+\frac{1}{p}\right)} \tau^{-\frac{m}{2}}\left(\frac{2}{\gamma}\right)^{m \frac{\ln 2}{\ln \gamma}\left(\frac{1}{q}-\frac{1}{2}\right)} 2^{-m \alpha \frac{\ln 2}{\ln \gamma}} \gamma^{m \frac{\ln 2}{\ln \gamma}\left(r+\frac{1}{q}-\frac{1}{p}\right)} \\
& =n^{\frac{1}{q}-\frac{1}{2}} \sum_{m} 2^{m\left(-\alpha \frac{\ln 2}{\ln \gamma}+\frac{1}{q}\right)} \tau^{-\frac{m}{2}}\left(\frac{2}{\gamma}\right)^{m \frac{\ln 2}{\ln \gamma}\left(\frac{1}{q}-\frac{1}{2}\right)} \stackrel{n}{\lesssim} n^{\frac{1}{q}-\frac{1}{2}} .
\end{aligned}
$$

- for $p \geq 2$, we estimate the sum over $m \leq k \frac{\ln \gamma}{\ln 2}$ :

$$
\begin{aligned}
& \sum_{k} \sum_{m \leq k \ln \gamma} d_{l_{k, m}}\left(B_{p}^{\nu_{k, m}}, l_{q}^{\nu_{k, m}}\right) 2^{-k \alpha} \underset{p, q, r}{\stackrel{4.18}{\lesssim}} \sum_{k} \sum_{m \leq k \frac{\ln \gamma}{\ln 2}} 2^{-k \alpha} n^{\frac{1}{q}-\frac{1}{p}}\left(\frac{2}{\gamma}\right)^{k\left(\frac{1}{q}-\frac{1}{p}\right)} 2^{\frac{m}{q} \lambda} \tau^{-\frac{m}{2} \lambda} \\
& \underset{p, q, \tau}{\lesssim} n^{\frac{1}{q}-\frac{1}{p}} \sum_{k} 2^{-k \alpha}\left(\frac{2}{\gamma}\right)^{k\left(\frac{1}{q}-\frac{1}{p}\right)} 2^{\frac{k}{q} \frac{\ln \gamma}{\ln 2} \lambda} \tau^{-\frac{k}{2} \frac{\ln \gamma}{\ln 2} \lambda} \stackrel{n}{\lesssim} n^{\frac{1}{q}-\frac{1}{p}}
\end{aligned}
$$

(because $\alpha>\frac{1}{q} \lambda$ ).

- for $p \geq 2$, we estimate the sum over $m>k \frac{\ln \gamma}{\ln 2}$ :

$$
\begin{aligned}
& \sum_{m} \sum_{k<m \frac{\ln 2}{\ln \gamma}} d_{l_{k, m}}\left(B_{p}^{\nu_{k, m}}, l_{q}^{\nu_{k, m}}\right) 2^{-k \alpha}\left(\frac{\gamma^{k}}{2^{m}}\right)^{r+\frac{1}{q}-\frac{1}{p}} \\
& \underset{p, q, r}{\stackrel{4.18}{\lesssim}} \sum_{m} \sum_{k<m \frac{\ln 2}{\ln \gamma}} 2^{-k \alpha} n^{\frac{1}{q}-\frac{1}{p}}\left(\frac{2}{\gamma}\right)^{k\left(\frac{1}{q}-\frac{1}{p}\right)} 2^{\frac{m}{q} \lambda} \tau^{-\frac{m}{2} \lambda} \gamma^{k\left(r+\frac{1}{q}-\frac{1}{p}\right)} 2^{-m\left(r+\frac{1}{q}-\frac{1}{p}\right)} \\
& \underset{p, q, r, \alpha, \gamma}{\lesssim} n^{\frac{1}{q}-\frac{1}{p}} \sum_{m} 2^{-\alpha m \frac{\ln 2}{\ln \gamma}}\left(\frac{2}{\gamma}\right)^{m \frac{\ln 2}{\ln \gamma}\left(\frac{1}{q}-\frac{1}{p}\right)} 2^{\frac{m}{q} \lambda} \tau^{-\frac{m}{2} \lambda} \gamma^{m\left(\log _{\gamma} 2\right)\left(r+\frac{1}{q}-\frac{1}{p}\right)} 2^{-m\left(r+\frac{1}{q}-\frac{1}{p}\right)} \\
& =n^{\frac{1}{q}-\frac{1}{p}} \sum_{m} 2^{m\left(-\alpha \frac{\ln 2}{\ln \gamma}+\frac{1}{q} \lambda\right)}\left(\frac{2}{\gamma}\right)^{m \frac{\ln 2}{\ln \gamma}\left(\frac{1}{q}-\frac{1}{p}\right)} \tau^{-\frac{m}{2} \lambda} \lesssim n^{\frac{1}{q}-\frac{1}{p}} .
\end{aligned}
$$

This completes the proof of (4.16).

Proof of the lower estimate. Let $x_{k}=e^{-k}, k=1, \ldots, n$, and let

$$
\begin{aligned}
& \tilde{g}(x)= \begin{cases}\min _{x \in\left[x_{k}, x_{k-1}\right]} g(x) & \text { if } x \in\left(x_{k}, x_{k-1}\right], \\
0 & \text { if } x \in\left[0, e^{-n}\right],\end{cases} \\
& \widetilde{v}(x)= \begin{cases}\min _{x \in\left[x_{k}, x_{k-1}\right]} v(x) & \text { if } x \in\left(x_{k}, x_{k-1}\right], \\
0 & \text { if } x \in\left[0, e^{-n}\right] .\end{cases}
\end{aligned}
$$

Then $\widehat{W}_{p, g}^{r}\left[0, e^{-1}\right] \supset \widehat{W}_{p, \tilde{g}}^{r}\left[0, e^{-1}\right]$ and $L_{q, v}\left[0, e^{-1}\right] \subset L_{q, \tilde{v}}\left[0, e^{-1}\right]$ (moreover, the embedding operators are continuous). Proposition 1 shows that $g(x) \underset{\beta_{g}, c_{*}}{\lesssim} \widetilde{g}(x)$ and $v(x) \underset{\beta_{g}, c_{*}}{\lesssim}$ 
$\widetilde{v}(x)$ for $x \in\left[e^{-n}, e^{-1}\right]$. Therefore,

$$
\int_{e^{-n}}^{e^{-1}} \widetilde{g}^{\varkappa}(x) \widetilde{v}^{\varkappa}(x) d x \gtrsim \int_{e^{-n}}^{e^{-1}} g^{\varkappa}(x) v^{\varkappa}(x) d x=\int_{1}^{n} \varphi_{g}^{\varkappa}(y) \varphi_{v}^{\varkappa}(y) d y .
$$

Applying this estimate and Lemma 2 for $N=2 n$, we obtain

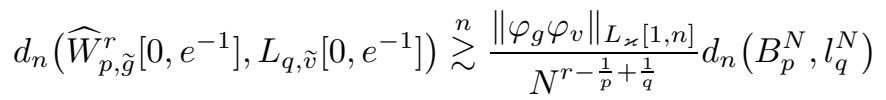

$$
\begin{aligned}
& 2.17,2.2,2.4 \\
& \underset{p, q, r}{\gtrsim}\left\|\varphi_{g} \varphi_{v}\right\|_{L_{\varkappa}[1, n]} d_{n}\left(W_{p}^{r}[0,1], L_{q}[0,1]\right) .
\end{aligned}
$$

The proof of the claim about approximation numbers is similar; in order to obtain the upper estimate, as $\Lambda_{k, m}$ and $E_{k, m}$ we take the extremal subspace and the linear operator for $\lambda_{l_{k, m}}\left(B_{p}^{\nu_{k, m}}, l_{q}^{\nu_{k, m}}\right)$ and then employ estimates (2.1), (2.3), and (2.5).

Example 1. Let $\alpha_{p q}$ be defined by (1.8), and let

$$
\varphi_{g}(y)=y^{-\alpha_{g}} \rho_{g}(y), \quad \varphi_{v}(y)=y^{-\alpha_{v}} \rho_{v}(y),
$$

where $\alpha:=\alpha_{g}+\alpha_{v}>\alpha_{p q}, \alpha \neq r+\frac{1}{q}-\frac{1}{p}$, and $\rho_{g}, \rho_{v}$ are positive absolutely continuous functions satisfying condition (1.9).

We check condition (\#). Consider the function $\varphi_{g}$ (for $\varphi_{v}$ the proof is similar). Let $\varepsilon>0$. Then the function $\varepsilon t+\ln \varphi_{g}(t)=\varepsilon t-\alpha_{g} \ln t+\ln \rho_{g}(t)$ is monotone increasing for sufficiently large $t$, because its derivative equals $\varepsilon-\frac{1}{t}\left(\alpha_{g}-\frac{t \rho_{g}^{\prime}(t)}{\rho_{g}(t)}\right)=\varepsilon+\underset{t \rightarrow+\infty}{o(1)}$. Similarly, $-\varepsilon t+\ln \varphi_{g}(t)$ is monotone decreasing for large $t$. Therefore, the functions

$$
\psi_{\varepsilon, \pm}(t)=\left\{\begin{array}{ll} 
\pm \varepsilon t+\ln \varphi_{g}(t) & \text { if } t \geq t_{\varepsilon}, \\
\pm \varepsilon t_{\varepsilon}+\ln \varphi_{g}\left(t_{\varepsilon}\right) & \text { if } 1 \leq t \leq t_{\varepsilon},
\end{array} \quad h_{\varepsilon, \pm}(t)= \pm \varepsilon t+\ln \varphi_{g}(t)-\psi_{\varepsilon, \pm}(t)\right.
$$

are as desired for an appropriate $t_{\varepsilon}>0$.

Now we check condition 2 of Theorem 1, Take $\mu \in\left(\alpha_{p q}, \alpha\right)$. We show that there exists $M=M(\mu)>0$ such that for any $c \geq 1, y \geq 1$ we have

$$
\varphi_{g}(c y) \varphi_{v}(c y) \leq M c^{-\mu} \varphi_{g}(y) \varphi_{v}(y),
$$

i.e., $\ln \rho_{g}(c y)+\ln \rho_{v}(c y) \leq \ln M+(\alpha-\mu) \ln c+\ln \rho_{g}(y)+\ln \rho_{v}(y)$. We choose $y_{1}$ so as to have $\frac{z \rho_{g}^{\prime}(z)}{\rho_{g}(z)}+\frac{z \rho_{v}^{\prime}(z)}{\rho_{v}(z)} \leq \alpha-\mu$ for $z \geq y_{1}$. Then for any $y \geq y_{1}$ we get

$$
\begin{gathered}
\ln \rho_{g}(c y)+\ln \rho_{v}(c y)=\ln \rho_{g}(y)+\ln \rho_{v}(y)+\int_{1}^{c} \frac{\rho_{g}^{\prime}(t y)}{\rho_{g}(t y)} y d t+\int_{1}^{c} \frac{\rho_{v}^{\prime}(t y)}{\rho_{v}(t y)} y d t \\
\leq \ln \rho_{g}(y)+\ln \rho_{v}(y)+\int_{1}^{c} \frac{\alpha-\mu}{t} d t=\ln \rho_{g}(y)+\ln \rho_{v}(y)+(\alpha-\mu) \ln c .
\end{gathered}
$$

If $y \leq c y \leq y_{1}$, then (4.19) follows from the boundedness of the function $\ln \varphi_{g}+\ln \varphi_{v}$ on $\left[1, y_{1}\right]$ and the inequality $c \leq y_{1}$. Let $y \leq y_{1} \leq c y, \widetilde{c}=c \frac{y}{y_{1}}$. Then, collecting the estimates already obtained, we get

$$
\begin{aligned}
\varphi_{g}(c y) \varphi_{v}(c y) & =\varphi_{g}\left(\widetilde{c} y_{1}\right) \varphi_{v}\left(\widetilde{c} y_{1}\right) \leq \widetilde{c}^{-\mu} \varphi_{g}\left(y_{1}\right) \varphi_{v}\left(y_{1}\right) \\
& \leq M\left(\frac{y_{1}}{y}\right)^{-\mu} \widetilde{c}^{-\mu} \varphi_{g}(y) \varphi_{v}(y)=M c^{-\mu} \varphi_{g}(y) \varphi_{v}(y)
\end{aligned}
$$


To check (1.5) in the case where $\alpha<r+\frac{1}{q}-\frac{1}{p}$, we denote $\rho(y)=\rho_{g}(y) \rho_{v}(y)$. Then $y \frac{\rho^{\prime}(y)}{\rho(y)} \underset{y \rightarrow+\infty}{\rightarrow} 0$. Let $y_{2}$ be such that

$$
-\frac{1}{2}<\frac{1}{\varkappa^{-1}-\alpha} y \frac{\rho^{\prime}(y)}{\rho(y)}<\frac{1}{2}
$$

for each $y \geq y_{2}$. Then for any $n \geq y_{2}$ we have

$$
\begin{aligned}
& (1-\alpha \varkappa) \ln n-(1-\alpha \varkappa) \ln y_{2}+\varkappa\left[\ln \rho(n)-\ln \rho\left(y_{2}\right)\right] \\
& =(1-\alpha \varkappa) \int_{y_{2}}^{n} \frac{d t}{t}+\varkappa \int_{y_{2}}^{n} \frac{t \rho^{\prime}(t)}{\rho(t)} \frac{d t}{t} \\
& \quad=(1-\alpha \varkappa) \int_{y_{2}}^{n}\left[1+\frac{1}{\varkappa^{-1}-\alpha} t \frac{\rho^{\prime}(t)}{\rho(t)}\right] \frac{d t}{t} \geq \frac{1-\alpha \varkappa}{2} \ln \frac{n}{y_{2}} .
\end{aligned}
$$

Consequently,

for all sufficiently large $n$, whence

$$
\frac{n^{1-\alpha \varkappa} \rho^{\varkappa}(n)}{y_{2}^{1-\alpha \varkappa} \rho^{\varkappa}\left(y_{2}\right)} \geq 2
$$

$$
\frac{1}{2} n^{-\alpha \varkappa} \rho^{\varkappa}(n) \leq n^{-\alpha \varkappa} \rho^{\varkappa}(n)-\frac{y_{2}^{1-\alpha \varkappa}}{n} \rho^{\varkappa}\left(y_{2}\right) \leq n^{-\alpha \varkappa} \rho^{\varkappa}(n) .
$$

Integration by parts yields the estimate

$$
\begin{aligned}
\frac{1}{n} \int_{y_{2}}^{n} & \varphi_{g}^{\varkappa}(y) \varphi_{v}^{\varkappa}(y) d y=\frac{1}{n} \int_{y_{2}}^{n} y^{-\alpha \varkappa} \rho^{\varkappa}(y) d y \\
& =\frac{1}{1-\alpha \varkappa}\left(n^{-\alpha \varkappa} \rho^{\varkappa}(n)-\frac{y_{2}^{1-\alpha \varkappa}}{n} \rho^{\varkappa}\left(y_{2}\right)\right)-\frac{1}{n(1-\alpha \varkappa)} \int_{y_{2}}^{n} \varkappa y^{1-\alpha \varkappa} \rho^{\varkappa-1}(y) \rho^{\prime}(y) d y \\
& \frac{4.20}{\leq} \frac{1}{2 n} \int_{y_{2}}^{n} y^{-\alpha \varkappa} \rho^{\varkappa}(y) d y+\frac{1}{1-\alpha \varkappa}\left(n^{-\alpha \varkappa} \rho^{\varkappa}(n)-\frac{y_{2}^{1-\alpha \varkappa}}{n} \rho^{\varkappa}\left(y_{2}\right)\right),
\end{aligned}
$$

along with a similar lower estimate; thus, by (4.21),

$$
n^{-r-\frac{1}{q}+\frac{1}{p}}\left\|\varphi_{g} \varphi_{v}\right\|_{L_{\varkappa}[1, n]} \stackrel{n}{\asymp}\left(\frac{1}{n} \int_{y_{2}}^{n} y^{-\alpha \varkappa} \rho^{\varkappa}(y) d y\right)^{r+\frac{1}{q}-\frac{1}{p}} \stackrel{n}{\asymp} n^{-\alpha} \rho(n) .
$$

Observe that, for any $\sigma>0$,

$$
y^{-\sigma} \rho(y) \downarrow 0, \quad y \rightarrow+\infty
$$

(the function is monotone decreasing for large $y$ ). Indeed, if $y$ is sufficiently large, then

$$
(-\sigma \ln y+\ln \rho(y))^{\prime}=-\frac{\sigma}{y}+\frac{\rho^{\prime}(y)}{\rho(y)}<-\frac{\sigma}{2 y} .
$$

Thus, $n^{-\alpha} \rho(n) \stackrel{n}{\asymp}\left\|\varphi_{g} \varphi_{v}\right\|_{L_{\infty}[n, \infty)}$, and (1.5) is proved for $q \geq p$. Let $q<p$. Then $y^{-\alpha+\frac{1}{q}-\frac{1}{p}} \rho(y) \rightarrow 0$ as $y \rightarrow \infty$ by (4.23). Hence,

$$
\begin{aligned}
\int_{n}^{\infty} & y^{-\alpha \frac{q p}{p-q}} \rho^{\frac{q p}{p-q}}(y) d y \\
& =\frac{1}{\alpha \frac{q p}{p-q}-1} n^{1-\alpha \frac{q p}{p-q}} \rho^{\frac{q p}{p-q}}(n)+\frac{1}{\alpha-\frac{1}{q}+\frac{1}{p}} \int_{n}^{\infty} y^{1-\alpha \frac{q p}{p-q}} \rho^{\frac{q p}{p-q}-1}(y) \rho^{\prime}(y) d y
\end{aligned}
$$

and for sufficiently large $n$ we have

$$
\left\|\varphi_{g} \varphi_{v}\right\|_{L_{s}[n, \infty)} \stackrel{n}{ } \rho(n) n^{-\alpha+\frac{1}{q}-\frac{1}{p}} .
$$


Thus, from (4.22) and Theorem 1 it follows that

$$
d_{n}\left(\widehat{W}_{p, g}^{r}\left[0, e^{-1}\right], L_{q, v}\left[0, e^{-1}\right]\right) \stackrel{n}{`} n^{-\alpha+r+\frac{1}{q}-\frac{1}{p}} \rho(n) d_{n}\left(W_{p}^{r}[0,1], L_{q}[0,1]\right)
$$

for $\alpha_{p q}<\alpha<r+\frac{1}{q}-\frac{1}{p}$.

Now we consider the case where $\alpha>r+\frac{1}{q}-\frac{1}{p}$. Then $g v \in L_{\varkappa}\left[0, e^{-1}\right]$. Indeed, suppose $\varkappa^{-1}<\mu<\alpha$. Notice that $\alpha_{p q}<r+\frac{1}{q}-\frac{1}{p}$. We have shown that (4.19) is valid for any $\mu \in\left(\alpha_{p q}, \alpha\right)$; therefore, $\varphi_{g}(y) \varphi_{v}(y) \stackrel{y}{\lesssim} y^{-\mu}$ whenever $\mu \in\left(\varkappa^{-1}, \alpha\right)$. Hence,

$$
\left\|\varphi_{g} \varphi_{v}\right\|_{L_{\varkappa}[1, n]} \stackrel{n}{=} 1
$$

On the other hand, for $p>q$ we have

$$
n^{r}\left\|\varphi_{g} \varphi_{v}\right\|_{L_{\frac{q p}{p-q}}[n,+\infty)} \stackrel{n}{\lesssim} n^{r}\left(\int_{n}^{\infty} y^{-\mu \frac{q p}{p-q}} d y\right)^{\frac{1}{q}-\frac{1}{p}} \underbrace{n} n^{r+\frac{1}{q}-\frac{1}{p}-\mu},
$$

and for $p \leq q$ we have

$$
n^{r+\frac{1}{q}-\frac{1}{p}}\left\|\varphi_{g} \varphi_{v}\right\|_{L_{\infty}[n,+\infty)} \stackrel{n}{\lesssim} n^{r+\frac{1}{q}-\frac{1}{p}-\mu}
$$

which implies that

$$
n^{\left(\frac{1}{p}-\frac{1}{q}\right)_{+}-r}\left\|\varphi_{g} \varphi_{v}\right\|_{L_{\varkappa}[1, n]} \geq\left\|\varphi_{g} \varphi_{v}\right\|_{L_{s}[n,+\infty)}
$$

for large $n$. This yields (1.5). Hence,

$$
d_{n}\left(\widehat{W}_{p, g}^{r}\left[0, e^{-1}\right], L_{q, v}\left[0, e^{-1}\right]\right) \stackrel{n}{`} d_{n}\left(W_{p}^{r}[0,1], L_{q}[0,1]\right) .
$$

Example 2. Let $\varphi_{g}(y)=y^{-\alpha_{g}} \rho_{g}(y), \varphi_{v}(y)=y^{-\alpha_{v}} \rho_{v}(y)$, where $\alpha_{g}+\alpha_{v}=r+\frac{1}{q}-\frac{1}{p}$, and $\rho_{g}, \rho_{v}$ are positive absolutely continuous functions satisfying (1.9), and let $\varphi_{g} \varphi_{v} \in$ $L_{\varkappa}[1,+\infty)$. We check condition (1.5). Let $\rho(y)=\rho_{g}(y) \rho_{v}(y)$. If $p>q$, then

$$
n^{r}\left(\int_{n}^{\infty} y^{-\varkappa^{-1} \frac{q p}{p-q}} \rho^{\frac{q p}{p-q}}(y) d y\right)^{\frac{1}{q}-\frac{1}{p}} \overbrace{}^{n} n^{r} \cdot \rho(n) n^{\frac{1}{q}-\frac{1}{p}-r-\frac{1}{q}+\frac{1}{p}}=\rho(n)
$$

(this can be proved in the same way as (4.24)), and if $p \leq q$, then, by (4.23),

$$
n^{r+\frac{1}{q}-\frac{1}{p}}\left\|\varphi_{g} \varphi_{v}\right\|_{L_{\infty}[n,+\infty)} \stackrel{n}{\lesssim} \rho(n)
$$

Thus, it remains to show that the sequence $\{\rho(n)\}$ is bounded. Indeed, (1.9) implies the existence of $y_{0} \geq 1$ such that $\rho^{\prime}(y) \leq \frac{\rho(y)}{y}$ for any $y \geq y_{0}$; therefore,

$$
\rho^{\varkappa}(n)-\rho^{\varkappa}\left(y_{0}\right)=\varkappa \int_{y_{0}}^{n} \rho^{\varkappa-1}(y) \rho^{\prime}(y) d y \leq \varkappa \int_{y_{0}}^{n} \frac{\rho^{\varkappa}(y)}{y} d y \leq \varkappa\left\|\varphi_{g} \varphi_{v}\right\|_{L_{\varkappa}\left[y_{0},+\infty\right)} .
$$

Conditions (\#) and (1.6) can be checked in the same way as in Example 1.

Example 3. Let $\varphi_{g}(y)=y^{-\alpha_{g}}, \varphi_{v}(y)=y^{-\alpha_{v}}$, and let $\alpha_{g}+\alpha_{v}=r+\frac{1}{q}-\frac{1}{p}$. Then

$$
\left\|\varphi_{g} \varphi_{v}\right\|_{L_{\varkappa}[1, n]}=(\ln n)^{r+\frac{1}{q}-\frac{1}{p}},\left\|\varphi_{g} \varphi_{v}\right\|_{L_{s}[n, \infty)} \stackrel{n}{=} n^{-r+\left(\frac{1}{p}-\frac{1}{q}\right)_{+}} .
$$

Hence, (1.5) is true, and

$$
\begin{aligned}
d_{n}\left(\widehat{W}_{p, g}^{r}\left[0, e^{-1}\right], L_{q, v}\left[0, e^{-1}\right]\right) & \stackrel{n}{ }\left\|\varphi_{g} \varphi_{v}\right\|_{L_{\varkappa}[1, n]} d_{n}\left(W_{p}^{r}[0,1], L_{q}[0,1]\right) \\
& =(\ln n)^{r+\frac{1}{q}-\frac{1}{p}} d_{n}\left(W_{p}^{r}[0,1], L_{q}[0,1]\right) .
\end{aligned}
$$


Proof of Theorem 2. We show that for any $\delta>0$ there exists $N \in \mathbb{N}$ such that for any $n \geq N$ and $\sigma \in \mathbb{R}_{+}$we have

$$
\left|\ln \rho\left(2^{\sigma} n\right)-\ln \rho(n)\right| \leq \sigma \cdot \delta \ln 2 .
$$

Indeed, if $n$ is sufficiently large, then

$$
\left|\ln \rho\left(2^{\sigma} n\right)-\ln \rho(n)\right| \leq \int_{n}^{2^{\sigma} n}\left|\frac{\rho^{\prime}(t)}{\rho(t)}\right| d t \leq \delta \int_{n}^{2^{\sigma} n} \frac{d t}{t}=\delta \ln \frac{2^{\sigma} n}{n}=\sigma \cdot \delta \ln 2 .
$$

Proof of the upper estimate. Set $k_{0}=\left[\left(\frac{q}{2}-1\right) \log _{2} n\right]$. By (4.25), we have

$$
\rho\left(2^{k_{0}} n\right) \stackrel{n}{=} \rho\left(n^{q / 2}\right) \text {. }
$$

For $m \in \mathbb{Z}_{+}$and $k=0, \ldots, k_{0}+1$, we define partitions $T_{k, m}$ and operators $P_{k, m}$ as follows. Put $T_{k_{0}+1, m}=\left\{\left[0, e^{-2^{k_{0}} n}\right]\right\}$; the objects $T_{0, m}, P_{0, m}$, and $P_{k_{0}+1,0}$ are defined in the same way as in the proof of Theorem 1 . Combining (4.3), the first inequality in (4.6), and (4.22), we get

$$
\begin{aligned}
& \left\|f-P_{0, m} f\right\|_{p, q, T_{0, m}, v} \stackrel{n, m}{\lesssim}\left(2^{m} n\right)^{-r-\frac{1}{q}+\frac{1}{p}}\left\|\varphi_{g} \varphi_{v}\right\|_{L_{\varkappa}[1, n]} \\
& \stackrel{n, m}{\asymp}\left(2^{m} n\right)^{-r-\frac{1}{q}+\frac{1}{p}} n^{r+\frac{1}{q}-\frac{1}{p}-\alpha} \rho(n)=2^{-\frac{m}{\varkappa}} n^{-\alpha} \rho(n) \text {, } \\
& \left\|f-P_{k_{0}+1,0} f\right\|_{L_{q, v}\left[0, e^{-2^{k_{0}}}\right]} \stackrel{n}{\lesssim}\left\|\varphi_{g} \varphi_{v}\right\|_{L_{\infty}\left[2^{k_{0}} n,+\infty\right)} \\
& \stackrel{n}{=} n^{-\alpha} 2^{-\alpha k_{0}} \rho\left(2^{k_{0}} n\right) \stackrel{n}{=} n^{-\frac{\alpha q}{2}} \rho\left(n^{q / 2}\right) .
\end{aligned}
$$

For any $k=1, \ldots, k_{0}$, we put $\lambda_{k}=\left(k_{0}-k+1\right)^{2}$. For each $m \in \mathbb{Z}_{+}$, consider the partition $T_{k, m}$ of $\left[e^{-2^{k} n}, e^{-2^{k-1} n}\right]$ by the points $e^{-2^{k-m} \lambda_{k} j}$. The number of such points equals $2^{m} \lambda_{k}^{-1} n$ up to a multiplicative constant. Then for any $m \geq 1$ the partition $T_{k, m}$ is a subpartition of $T_{k, m-1}$, and $T_{k, m-1}$ contains at most two segments from $T_{k, m}$. Set $\left(P_{k, m} f\right)(x)=0$ for $x \notin\left[e^{-2^{k} n}, e^{-2^{k-1} n}\right],\left(P_{k, m} f\right)(x)=\left(P^{\sigma, \tau} f\right)(x)$ if $[\sigma, \tau] \in T_{k, m}$ (the $P^{\sigma, \tau} f$ are defined as in the proof of Theorem 11). By Proposition 4 and (4.25),

$$
\begin{aligned}
\left\|P_{k, m} f-P_{k, m-1} f\right\|_{p, q, T_{k, m}, v} & \stackrel{n, m, k}{\lesssim}\left\|\varphi_{g} \varphi_{v}\right\|_{L_{\infty}\left[2^{k-1} n, \infty\right)} \min \left\{1,\left(2^{k-m} \lambda_{k}\right)^{r+\frac{1}{q}-\frac{1}{p}}\right\} \\
& \asymp 2^{-\alpha k} n^{-\alpha} \rho\left(2^{k} n\right) \min \left\{1,\left(2^{k-m} \lambda_{k}\right)^{r+\frac{1}{q}-\frac{1}{p}}\right\} .
\end{aligned}
$$

Hence, formula (4.12) is valid, where the sum is taken in $L_{q, v}$. We define $P f$ by formula (4.15); $A_{k, m}, \nu_{k, m}$, and $E_{k, m}$ are defined in the same way as in Theorem 1 . Then the image of $P$ is contained in the subspace $V$, and

$$
\begin{aligned}
& \operatorname{dim} V \leq r \widehat{n}+r \sum_{k=1}^{k_{0}}\left(\lambda_{k}^{-1} n+1\right)+r+\sum_{m \in \mathbb{N}} \sum_{0 \leq k \leq k_{0}} l_{k, m} \stackrel{n}{\lesssim} n, \\
& \|f-P f\|_{q, v} \leq\left\|f-P_{k_{0}+1,0} f\right\|_{L_{q, v}\left[0, e^{-2^{k_{0}}}\right]} \\
& +\sum_{m \in \mathbb{N}, 0 \leq k \leq k_{0}}\left\|P_{k, m} f-P_{k, m-1} f\right\|_{p, q, T_{k, m}, v} d_{l_{k, m}}\left(B_{p}^{\nu_{k, m}}, l_{q}^{\nu_{k, m}}\right) \\
& \text { 4.26), 4.27, } 4.28 \\
& \stackrel{n, m, k}{\lesssim} n^{-\frac{\alpha q}{2}} \rho\left(n^{q / 2}\right)+n^{-\alpha} \rho(n) \sum_{m \in \mathbb{N}} 2^{-m\left(r+\frac{1}{q}-\frac{1}{p}\right)} d_{l_{0, m}}\left(B_{p}^{\nu_{0, m}}, l_{q}^{\nu_{0, m}}\right) \\
& +\sum_{1 \leq k \leq k_{0}, m \in \mathbb{N}} 2^{-\alpha k} n^{-\alpha} \rho\left(2^{k} n\right) \min \left\{1,\left(2^{k-m} \lambda_{k}\right)^{r+\frac{1}{q}-\frac{1}{p}}\right\} d_{l_{k, m}}\left(B_{p}^{\nu_{k, m}}, l_{q}^{\nu_{k, m}}\right) .
\end{aligned}
$$


By Lemma 1, there exists a sequence $l_{0, m}$ such that

$$
\sum_{m} l_{0, m} \underset{p, q, r}{\lesssim} n \text { and } \sum_{m \in \mathbb{N}} 2^{-m\left(r+\frac{1}{q}-\frac{1}{p}\right)} d_{l_{0, m}}\left(B_{p}^{\nu_{0, m}}, l_{q}^{\nu_{0, m}}\right) \underset{p, q, r}{\lesssim} n^{\frac{1}{q}-\min \left\{\frac{1}{2}, \frac{1}{p}\right\}}
$$

which implies

$$
\begin{aligned}
& n^{-\alpha} \rho(n) \sum_{m \in \mathbb{N}} 2^{-m\left(r+\frac{1}{q}-\frac{1}{p}\right)} d_{l_{0, m}}\left(B_{p}^{\nu_{0, m}}, l_{q}^{\nu_{0, m}}\right) \\
& \quad \underset{p, q, r}{\quad} n^{-\alpha+\frac{1}{q}-\min \left\{\frac{1}{2}, \frac{1}{p}\right\}} \rho(n) \stackrel{n}{\lesssim} n^{-\alpha+\frac{1}{q}-\min \left\{\frac{1}{2}, \frac{1}{p}\right\}} 2^{k_{0} \delta} \rho\left(2^{k_{0}} n\right) \stackrel{n}{\lesssim} n^{-\frac{\alpha q}{2}} \rho\left(n^{q / 2}\right)
\end{aligned}
$$

(the penultimate inequality follows from (4.25), and $\delta>0$ is such that $-\alpha+\frac{1}{q}-$ $\left.\min \left\{\frac{1}{2}, \frac{1}{p}\right\}+\delta\left(\frac{q}{2}-1\right)<-\frac{\alpha q}{2}\right)$.

Consider $1 \leq k \leq k_{0}$. Let $m_{0}=k_{0}$, let $\mu_{m}=\left(m_{0}-m+1\right)^{-2}$ for $m \leq m_{0}, l_{k, m}=0$ for $m \geq m_{0}$, and let $l_{k, m}=\left[\mu_{m} \lambda_{k}^{-1} n\right]$ for $m<m_{0}$. Then

$$
\begin{aligned}
& \sum_{1 \leq k \leq k_{0}} \sum_{m \geq m_{0}} 2^{-\alpha k} n^{-\alpha} \rho\left(2^{k} n\right) \min \left\{1,\left(2^{k-m} \lambda_{k}\right)^{r+\frac{1}{q}-\frac{1}{p}}\right\} d_{l_{k, m}}\left(B_{p}^{\nu_{k, m}}, l_{q}^{\nu_{k, m}}\right) \\
& \leq \sum_{m \geq m_{0}} \sum_{k=1}^{k_{0}} 2^{-\alpha k} n^{-\alpha} \rho\left(2^{k} n\right)\left(2^{k-m} \lambda_{k}\right)^{r+\frac{1}{q}-\frac{1}{p}} \\
& =\sum_{m \geq m_{0}} n^{-\alpha} \rho\left(2^{k_{0}} n\right) 2^{\left(r+\frac{1}{q}-\frac{1}{p}-\alpha\right) k_{0}} 2^{-\left(r+\frac{1}{q}-\frac{1}{p}\right) m} \\
& \times \sum_{k=1}^{k_{0}} \frac{\rho\left(2^{k} n\right)}{\rho\left(2^{k_{0}} n\right)} 2^{-\left(r+\frac{1}{q}-\frac{1}{p}-\alpha\right)\left(k_{0}-k\right)}\left(k_{0}-k+1\right)^{2\left(r+\frac{1}{q}-\frac{1}{p}\right)} \\
& 4.25 \\
& \stackrel{n}{\lesssim} \sum_{m \geq m_{0}} n^{-\alpha} \rho\left(n^{q / 2}\right) 2^{\left(r+\frac{1}{q}-\frac{1}{p}-\alpha\right) m_{0}} 2^{-\left(r+\frac{1}{q}-\frac{1}{p}\right) m} \\
& \underset{p, q, r}{\lesssim} n^{-\alpha} \rho\left(n^{q / 2}\right) 2^{-\alpha m_{0}} \stackrel{n}{=} n^{-\frac{\alpha q}{2}} \rho\left(n^{q / 2}\right) .
\end{aligned}
$$

It remains to estimate the quantity

$$
\sum_{1 \leq k \leq k_{0}} \sum_{1 \leq m \leq m_{0}} 2^{-\alpha k} n^{-\alpha} \rho\left(2^{k} n\right) \min \left\{1,\left(2^{k-m} \lambda_{k}\right)^{r+\frac{1}{q}-\frac{1}{p}}\right\} d_{l_{k, m}}\left(B_{p}^{\nu_{k, m}}, l_{q}^{\nu_{k, m}}\right),
$$

by applying (2.2), setting $\lambda=\frac{\frac{1}{p}-\frac{1}{q}}{\frac{1}{2}-\frac{1}{q}}$, and using the fact that $\nu_{k, m} \stackrel{n, m}{=}, k 2^{m} n \lambda_{k}^{-1}$.

- For $p<2$, we estimate the sum over $k \geq m$ :

$$
\begin{aligned}
& \sum_{k=1}^{k_{0}} \sum_{m=1}^{k} 2^{-\alpha k} n^{-\alpha} \rho\left(2^{k} n\right) d_{l_{k, m}}\left(B_{p}^{\nu_{k, m}}, l_{q}^{\nu_{k, m}}\right) \\
& \quad \sum_{k=1}^{n} \sum_{m=1}^{k_{0}} 2^{-\alpha k} n^{-\alpha} \rho\left(2^{k} n\right) 2^{\frac{m}{q}} n^{\frac{1}{q}-\frac{1}{2}} \lambda_{k}^{\frac{1}{2}-\frac{1}{q}} \mu_{m}^{-\frac{1}{2}} \\
& \quad=\sum_{k=1}^{k_{0}} 2^{-\alpha k} \lambda_{k}^{\frac{1}{2}-\frac{1}{q}} n^{-\alpha+\frac{1}{q}-\frac{1}{2}} \rho\left(2^{k} n\right) \sum_{m=1}^{k} 2^{\frac{m}{q}}\left(m_{0}-m+1\right) \\
& \quad=\sum_{k=1}^{k_{0}} 2^{-\alpha k} \lambda_{k}^{\frac{1}{2}-\frac{1}{q}} n^{-\alpha+\frac{1}{q}-\frac{1}{2}} \rho\left(2^{k} n\right) 2^{\frac{k}{q}}\left(m_{0}-k+1\right) \sum_{l=0}^{k-1} 2^{-\frac{l}{q}}\left(1+\frac{l}{m_{0}-k+1}\right)
\end{aligned}
$$




$$
\begin{aligned}
& \underset{q}{\lesssim} \sum_{k=1}^{k_{0}} n^{-\alpha+\frac{1}{q}-\frac{1}{2}} 2^{\left(\frac{1}{q}-\alpha\right) k}\left(k_{0}-k+1\right)^{1-\frac{2}{q}}\left(k_{0}-k+1\right) \rho\left(2^{k} n\right) \\
& =n^{-\alpha+\frac{1}{q}-\frac{1}{2}} 2^{\left(\frac{1}{q}-\alpha\right) k_{0}} \rho\left(2^{k_{0}} n\right) \sum_{k=1}^{k_{0}} 2^{-\left(\frac{1}{q}-\alpha\right)\left(k_{0}-k\right)} \frac{\rho\left(2^{k} n\right)}{\rho\left(2^{k_{0}} n\right)}\left(k_{0}-k+1\right)^{2-\frac{2}{q}} \\
& \stackrel{4.25}{\underbrace{n}} n^{-\alpha+\frac{1}{q}-\frac{1}{2}} n^{\left(-\alpha+\frac{1}{q}\right)\left(\frac{q}{2}-1\right)} \rho\left(n^{q / 2}\right)=n^{-\frac{\alpha q}{2}} \rho\left(n^{q / 2}\right) .
\end{aligned}
$$

- For $p<2$, we estimate the sum over $m \geq k$ :

$$
\begin{aligned}
\sum_{m=1}^{m_{0}} & \sum_{k=1}^{m} 2^{-\alpha k} n^{-\alpha} \rho\left(2^{k} n\right)\left(2^{k-m} \lambda_{k}\right)^{r+\frac{1}{q}-\frac{1}{p}} d_{l_{k, m}}\left(B_{p}^{\nu_{k, m}}, l_{q}^{\nu_{k, m}}\right) \\
& \lesssim \sum_{m=1}^{n} \sum_{k=1}^{m_{0}} 2^{-\alpha k} n^{-\alpha} \rho\left(2^{k} n\right)\left(2^{k-m} \lambda_{k}\right)^{r+\frac{1}{q}-\frac{1}{p}} 2^{\frac{m}{q}} n^{\frac{1}{q}-\frac{1}{2}} \lambda_{k}^{\frac{1}{2}-\frac{1}{q}} \mu_{m}^{-\frac{1}{2}} \\
& =\sum_{m=1}^{m_{0}} n^{-\alpha+\frac{1}{q}-\frac{1}{2}} 2^{-m\left(r-\frac{1}{p}\right)} \mu_{m}^{-\frac{1}{2}} \sum_{k=1}^{m} \rho\left(2^{k} n\right) 2^{k\left(r+\frac{1}{q}-\frac{1}{p}-\alpha\right)}\left(k_{0}-k+1\right)^{2 r+1-\frac{2}{p}} \\
& =\sum_{m=1}^{m_{0}} n^{-\alpha+\frac{1}{q}-\frac{1}{2}} 2^{m\left(\frac{1}{q}-\alpha\right)}\left(m_{0}-m+1\right)^{2 r+2-\frac{2}{p}} \rho\left(2^{m} n\right) \\
& \times \sum_{l=0}^{m-1} \frac{\rho\left(2^{m-l} n\right)}{\rho\left(2^{m} n\right)} 2^{-l\left(r+\frac{1}{q}-\frac{1}{p}-\alpha\right)}\left(1+\frac{l}{m_{0}-m+1}\right)^{2 r+1-\frac{2}{p}} \\
& \quad \sum_{m=1}^{n} n^{-\alpha+\frac{1}{q}-\frac{1}{2}} 2^{m\left(\frac{1}{q}-\alpha\right)}\left(m_{0}-m+1\right)^{2 r+2-\frac{2}{p}} \rho\left(2^{m} n\right) \\
& \vdots n^{-\alpha+\frac{1}{q}-\frac{1}{2}} 2^{m_{0}\left(\frac{1}{q}-\alpha\right)} \rho\left(2^{m_{0}} n\right) \sum_{m=1}^{m_{0}} 2^{-\left(m_{0}-m\right)\left(\frac{1}{q}-\alpha\right)}\left(m_{0}-m+1\right)^{2 r+2-\frac{2}{p}} \frac{\rho\left(2^{m} n\right)}{\rho\left(2^{m_{0}} n\right)} \\
& \frac{4.25}{{ }^{n}} \\
& \lesssim n^{-\frac{\alpha q}{2}} \rho\left(n^{q / 2}\right) .
\end{aligned}
$$

- For $p \geq 2$, we estimate the sum over $k \geq m$ :

$$
\begin{aligned}
& \sum_{k=1}^{k_{0}} \sum_{m=1}^{k} 2^{-\alpha k} n^{-\alpha} \rho\left(2^{k} n\right) d_{l_{k, m}}\left(B_{p}^{\nu_{k, m}}, l_{q}^{\nu_{k, m}}\right) \\
& \quad \sum_{k=1}^{n} \sum_{m=1}^{k_{0}} n^{-\alpha} 2^{-\alpha k} \rho\left(2^{k} n\right) n^{\frac{1}{q}-\frac{1}{p}} 2^{\frac{m}{q} \lambda} \lambda_{k}^{\frac{1}{p}-\frac{1}{q}} \mu_{m}^{-\frac{\lambda}{2}} \\
& \quad=\sum_{k=1}^{k_{0}} n^{-\alpha+\frac{1}{q}-\frac{1}{p}} 2^{-\alpha k} \lambda_{k}^{\frac{1}{p}-\frac{1}{q}} \rho\left(2^{k} n\right) \sum_{m=1}^{k} 2^{\frac{m \lambda}{q}}\left(m_{0}-m+1\right)^{\lambda} \\
& \quad \underset{p, q}{\sum_{k=1}^{n}} n^{-\alpha+\frac{1}{q}-\frac{1}{p}} \rho\left(2^{k} n\right) 2^{\left(\frac{\lambda}{q}-\alpha\right) k}\left(k_{0}-k+1\right)^{\lambda\left(2-\frac{2}{q}\right)} \\
& \quad n^{-\alpha+\frac{1}{q}-\frac{1}{p}} 2^{\left(\frac{\lambda}{q}-\alpha\right) k_{0}} \rho\left(n^{q / 2}\right) \stackrel{n}{`} n^{-\frac{\alpha q}{2}} \rho\left(n^{q / 2}\right) .
\end{aligned}
$$


- For $p \geq 2$, we estimate the sum over $k \leq m$ :

$$
\begin{aligned}
\sum_{m=1}^{m_{0}} & \sum_{k=1}^{m} 2^{-\alpha k} n^{-\alpha} \rho\left(2^{k} n\right)\left(2^{k-m} \lambda_{k}\right)^{r+\frac{1}{q}-\frac{1}{p}} d_{l_{k, m}}\left(B_{p}^{\nu_{k, m}}, l_{q}^{\nu_{k, m}}\right) \\
& \lesssim \sum_{m=1}^{n} \sum_{k=1}^{m_{0}} n^{-\alpha} 2^{-\alpha k} \rho\left(2^{k} n\right)\left(2^{k-m} \lambda_{k}\right)^{r+\frac{1}{q}-\frac{1}{p}} n^{\frac{1}{q}-\frac{1}{p}} 2^{\frac{m}{q} \lambda} \lambda_{k}^{\frac{1}{p}-\frac{1}{q}} \mu_{m}^{-\frac{\lambda}{2}} \\
& =\sum_{m=1}^{m_{0}} n^{-\alpha+\frac{1}{q}-\frac{1}{p}} 2^{m\left(\frac{\lambda}{q}-r-\frac{1}{q}+\frac{1}{p}\right)} \mu_{m}^{-\frac{\lambda}{2}} \sum_{k=1}^{m} \rho\left(2^{k} n\right) 2^{k\left(r+\frac{1}{q}-\frac{1}{p}-\alpha\right)} \lambda_{k}^{r} \\
& \lesssim \sum_{m=1}^{n} n^{-\alpha+\frac{1}{q}-\frac{1}{p}} \rho\left(2^{m} n\right) 2^{m\left(\frac{\lambda}{q}-r-\frac{1}{q}+\frac{1}{p}\right)} 2^{m\left(r+\frac{1}{q}-\frac{1}{p}-\alpha\right)}\left(m_{0}-m+1\right)^{2 r+\lambda} \\
& \lesssim n^{-\alpha+\frac{1}{q}-\frac{1}{p}} \rho\left(n^{q / 2}\right) 2^{m_{0}\left(\frac{\lambda}{q}-\alpha\right)} \stackrel{n}{`} n^{-\alpha+\frac{1}{q}-\frac{1}{p}+\left(\frac{q}{2}-1\right)\left(\frac{\lambda}{q}-\alpha\right)} \rho\left(n^{q / 2}\right) \stackrel{n}{`} n^{-\frac{\alpha q}{2}} \rho\left(n^{q / 2}\right) .
\end{aligned}
$$

Proof of the lower estimate. Arguing as in Theorem 1, we can prove that, for any $k \in \mathbb{Z}_{+}$,

$$
\begin{gathered}
d_{n}\left(\widehat{W}_{p, g}^{r}\left[0, e^{-1}\right], L_{q, v}\left[0, e^{-1}\right]\right) \stackrel{n, k}{\underset{n}{\sim}\left\|\varphi_{g} \varphi_{v}\right\|_{L_{\varkappa}\left[1,2^{k} n\right]}\left(2^{k} n\right)^{-r-\frac{1}{q}+\frac{1}{p}}} d_{n}\left(B_{p}^{2^{k} n}, l_{q}^{2^{k} n}\right) \\
\stackrel{n, k}{\rightleftharpoons}\left(2^{k} n\right)^{-\alpha} \rho\left(2^{k} n\right) d_{n}\left(B_{p}^{2^{k} n}, l_{q}^{2^{k} n}\right)
\end{gathered}
$$

by (4.22). If $p<2$, then from (2.2) it follows that

$$
d_{n}\left(B_{p}^{2^{k} n}, l_{q}^{2^{k} n}\right) \underset{p, q}{\asymp} \min \left\{2^{\frac{k}{q}} n^{\frac{1}{q}-\frac{1}{2}}, 1\right\} .
$$

Hence, if $2^{\frac{k}{q}} \leq n^{\frac{1}{2}-\frac{1}{q}}$, then

$$
\left(2^{k} n\right)^{-\alpha} \rho\left(2^{k} n\right) d_{n}\left(B_{p}^{2^{k} n}, l_{q}^{2^{k} n}\right) \underset{p, q}{\asymp} 2^{k\left(\frac{1}{q}-\alpha\right)} n^{-\alpha+\frac{1}{q}-\frac{1}{2}} \rho\left(2^{k} n\right) .
$$

Setting $k=k_{0}=\left[\left(\frac{q}{2}-1\right) \log _{2} n\right]$, we get

$$
\begin{aligned}
& d_{n}\left(\widehat{W}_{p, g}^{r}\left[0, e^{-1}\right], L_{q, v}\left[0, e^{-1}\right]\right) \gtrsim 2^{k_{0}\left(\frac{1}{q}-\alpha\right)} n^{-\alpha+\frac{1}{q}-\frac{1}{2}} \rho\left(2^{k_{0}} n\right) \\
& \underset{q, \alpha}{4.25} n^{\left(\frac{q}{2}-1\right)\left(\frac{1}{q}-\alpha\right)-\alpha+\frac{1}{q}-\frac{1}{2}} \rho\left(n^{q / 2}\right)=n^{-\frac{\alpha q}{2}} \rho\left(n^{q / 2}\right) \text {. }
\end{aligned}
$$

If $p \geq 2$, then $d_{n}\left(B_{p}^{2^{k} n}, l_{q}^{2^{k} n}\right) \underset{p, q}{\asymp} \min \left\{n^{\frac{1}{q}-\frac{1}{p}} 2^{\frac{k \lambda}{q}}, 1\right\}$, where $\lambda=\frac{\frac{1}{p}-\frac{1}{q}}{\frac{1}{2}-\frac{1}{q}}$. Therefore, for $k \leq k_{0}$ we have

$$
\left(2^{k} n\right)^{-\alpha} \rho\left(2^{k} n\right) d_{n}\left(B_{p}^{2^{k} n}, l_{q}^{2^{k} n}\right) \underset{p, q}{\asymp} n^{-\alpha+\frac{1}{q}-\frac{1}{p}} 2^{k\left(\frac{\lambda}{q}-\alpha\right)} \rho\left(2^{k} n\right),
$$

which implies

$$
\begin{aligned}
d_{n}\left(\widehat{W}_{p, g}^{r}\left[0, e^{-1}\right], L_{q, v}\left[0, e^{-1}\right]\right) & \stackrel{n}{ }{ }^{n} n^{-\alpha+\frac{1}{q}-\frac{1}{p}} 2^{k_{0}\left(\frac{\lambda}{q}-\alpha\right)} \rho\left(2^{k_{0}} n\right) \\
& \widetilde{\widetilde{q}, \alpha} n^{-\alpha+\frac{1}{q}-\frac{1}{p}+\left(\frac{1}{2}-\frac{1}{q}\right)(\lambda-\alpha q)} \rho\left(n^{q / 2}\right)=n^{-\frac{\alpha q}{2}} \rho\left(n^{q / 2}\right) .
\end{aligned}
$$

Remark. Similar assertions can be proved for function classes on the half-axis $[e,+\infty)$ (conditions on the functions $g, v$ and the asymptotics of the widths look similarly; to construct the required partition, we take the points $e^{\sigma}$ instead of $e^{-\sigma}$ ). 
§5. Appendix: proofs of the Auxiliary assertions

Proposition 5. Suppose $\rho>0, \nu \in \mathbb{R} \backslash\{0\}$, and $0 \leq \sigma<\tau \leq e^{-1}$. Let $\varphi$ satisfy (\#). If $\nu>0$, then

$$
\int_{\sigma}^{\tau} t^{-1+\nu} \varphi^{\rho}(|\ln t|) d t \underset{\nu, \rho, c_{\varphi}}{\lesssim} \tau^{\nu} \varphi^{\rho}(|\ln \tau|)
$$

and if $\nu<0$, then

$$
\int_{\sigma}^{\tau} t^{-1+\nu} \varphi^{\rho}(|\ln t|) d t \underset{\nu, \rho, c_{\varphi}}{\lesssim} \sigma^{\nu} \varphi^{\rho}(|\ln \sigma|)
$$

Proof. We prove (5.1); (5.2) is proved similarly. Since $\nu>0$, for any $y \in[|\ln \tau|,|\ln \sigma|]$ we have

$-\frac{\nu}{2} y+\rho \ln \varphi(y)=\rho\left(\psi_{\frac{\nu}{2 \rho},-}(y)+h_{\frac{\nu}{2 \rho},-}(y)\right) \leq \rho\left(\psi_{\frac{\nu}{2 \rho},-}(|\ln \tau|)+h_{\frac{\nu}{2 \rho},-}(|\ln \tau|)+2 c_{\varphi}\left(\frac{\nu}{2 \rho}\right)\right)$.

Hence,

$$
\begin{gathered}
\int_{\sigma}^{\tau} t^{-1+\nu} \varphi^{\rho}(|\ln t|) d t=\int_{\sigma}^{\tau} e^{-\nu|\ln t|+\rho \ln \varphi(|\ln t|)} d \ln t=\int_{|\ln \tau|}^{|\ln \sigma|} e^{-\frac{\nu}{2} y} e^{-\frac{\nu}{2} y+\rho \ln \varphi(y)} d y \\
\underset{\nu, \rho, c_{\varphi}}{\lesssim} e^{-\frac{\nu}{2}|\ln \tau|+\rho \ln \varphi(|\ln \tau|)} \int_{|\ln \tau|}^{|\ln \sigma|} e^{-\frac{\nu}{2} y} d y \underset{\nu}{\lesssim} e^{\nu \ln \tau+\rho \ln \varphi(|\ln \tau|)}=\tau^{\nu} \varphi^{\rho}(|\ln \tau|) .
\end{gathered}
$$

Proof of Proposition 1. It suffices to prove the claim for the function $g$. We may assume that $\sigma<\tau$. Since $a \leq b \leq e a$, we have $\frac{\tau^{-\beta_{g}}}{\sigma^{-\beta_{g}}} \underset{\widehat{\beta}_{g}}{\asymp}$. We estimate $\frac{\varphi_{g}(|\ln \tau|)}{\varphi_{g}(|\ln \sigma|)}$ from above (the estimate from below can be obtained similarly). We have

$$
\begin{aligned}
& \ln \varphi_{g}(|\ln \tau|)-\ln \varphi_{g}(|\ln \sigma|) \\
& \quad=(|\ln \sigma|-|\ln \tau|)+\left(|\ln \tau|+\ln \varphi_{g}(|\ln \tau|)\right)-\left(|\ln \sigma|+\ln \varphi_{g}(|\ln \sigma|)\right) \\
& \quad \leq 1+\psi_{1,+}(|\ln \tau|)+h_{1,+}(|\ln \tau|)-\psi_{1,+}(|\ln \sigma|)-h_{1,+}(|\ln \sigma|) \\
& \quad \leq 1+2 c_{*}(1) .
\end{aligned}
$$

Proof of Proposition 2, The first inequality follows from Proposition 1 and the simple estimate

$$
\max _{0 \leq k \leq r}\left\|\widetilde{I}_{r, g, v}^{a, b, k}\right\|_{L_{p}[a, b] \rightarrow L_{q}[a, b]} \underset{p, q, r}{\lesssim}\|g\|_{L_{p^{\prime}}[a, b]}\|v\|_{L_{q}[a, b]}(b-a)^{r-1}
$$

(see Theorems $\mathrm{A}$ and $\mathrm{B}$ ). The second inequality is true because

$$
\begin{aligned}
\|g v\|_{L_{\varkappa}[a, b]}^{\varkappa} & =\int_{a}^{b} x^{-1} \varphi_{g}^{\varkappa}(|\ln x|) \varphi_{v}^{\varkappa}(|\ln x|) d x \\
& =\int_{|\ln b|}^{|\ln a|} \varphi_{g}^{\varkappa}(y) \varphi_{v}^{\varkappa}(y) d y \leq\left\|\varphi_{g} \varphi_{v}\right\|_{L_{\infty}[|\ln b|,|\ln a|]}^{\varkappa} \ln \frac{b}{a} .
\end{aligned}
$$

Proof of Proposition 3, Let $\beta_{v}<\frac{1}{q}$. Then $\beta_{g}>r-\frac{1}{p}$. 
We estimate $A_{0}$ and $A_{1}$ :

$$
\begin{aligned}
& A_{0}^{\frac{q p}{p-q}}=\int_{a}^{b}\left(\int_{a}^{t}(t-x)^{q(r-1)} x^{-\beta_{v} q} \varphi_{v}^{q}(|\ln x|) d x\right)^{\frac{p}{p-q}} \\
& \times\left(\int_{t}^{b} x^{-p^{\prime} \beta_{g}} \varphi_{g}^{p^{\prime}}(|\ln x|) d x\right)^{\frac{p(q-1)}{p-q}} t^{-p^{\prime} \beta_{g}} \varphi_{g}^{p^{\prime}}(|\ln t|) d t \\
& \underset{p, q, r, \beta_{g}, c_{*}}{\underset{5.1}{(5.2)}} \int_{a}^{b} t^{\frac{p q}{p-q}(r-1)} \varphi_{v}^{\frac{p q}{p-q}}(|\ln t|) t^{\left(-\beta_{v} q+1\right) \frac{p}{p-q}} t^{\left(1-p^{\prime} \beta_{g}\right) \frac{q(p-1)}{p-q}} t^{-1} \varphi_{g}^{\frac{q p}{p-q}}(|\ln t|) d t \\
& =\int_{a}^{b} \varphi_{v}^{\frac{p q}{p-q}}(|\ln t|) \varphi_{g}^{\frac{q p}{p-q}}(|\ln t|) t^{-1} d t=\int_{|\ln b|}^{|\ln a|} \varphi_{g}^{\frac{q p}{p-q}}(y) \varphi_{v}^{\frac{q p}{p-q}}(y) d y, \\
& A_{1}^{\frac{q p}{p-q}}=\int_{a}^{b}\left(\int_{a}^{t} x^{-q \beta_{v}} \varphi_{v}^{q}(|\ln x|) d x\right)^{\frac{q}{p-q}} \\
& \times\left(\int_{t}^{b}(x-t)^{\frac{p}{p-1}(r-1)} x^{-\frac{p}{p-1} \beta_{g}} \varphi_{g}^{\frac{p}{p-1}}(|\ln x|) d x\right)^{\frac{q(p-1)}{p-q}} t^{-\beta_{v} q} \varphi_{v}^{q}(|\ln t|) d t \\
& \underset{p, q, r, \beta_{g}, c_{*}}{\stackrel{5.11}{\underset{15.2}{\infty}}} \int_{a}^{b} t^{-1} \varphi_{g}^{\frac{q p}{p-q}}(|\ln t|) \varphi_{v}^{\frac{q p}{p-q}}(|\ln t|) d t=\int_{|\ln b|}^{|\ln a|} \varphi_{g}^{\frac{q p}{p-q}}(y) \varphi_{v}^{\frac{q p}{p-q}}(y) d y .
\end{aligned}
$$

In the case where $\beta_{v}>r-1+\frac{1}{q}$ the proof is similar.

Suppose $k-1+\frac{1}{q}<\beta_{v}<k+\frac{1}{q}, k \in\{1, \ldots, r-1\}$. We estimate $A_{0, k}$ and $A_{1, k}$ :

$$
\begin{aligned}
& A_{0, k}^{\frac{p q}{p-q}} \leq \int_{a}^{b}\left(\int_{t}^{b} \varphi_{v}^{q}(|\ln x|) x^{\left(-\beta_{v}+k-1\right) q} d x\right)^{\frac{p}{p-q}} \\
& \times\left(\int_{a}^{t} \varphi_{g}^{p^{\prime}}(|\ln x|) x^{\left(-\beta_{g}+r-k\right) p^{\prime}} d x\right)^{\frac{p(q-1)}{p-q}} \varphi_{g}^{p^{\prime}}(|\ln t|) t^{\left(-\beta_{g}+r-k\right) p^{\prime}} d t \\
& \underset{p, q, r, \beta_{g}, c_{*}}{\stackrel{5.10}{[5.2}} \int_{a}^{b} \varphi_{v}^{\frac{p q}{p-q}}(|\ln t|) t^{\left(-\beta_{v}+k-1+\frac{1}{q}\right) \frac{p q}{p-q}} t^{\left(-\beta_{g}+r-k+\frac{1}{p^{\prime}}\right) \frac{p q}{p-q}-1} \varphi_{g}^{\frac{p q}{p-q}}(|\ln t|) d t \\
& \leq \int_{a}^{b} \varphi_{v}^{\frac{p q}{p-q}}(|\ln t|) \varphi_{g}^{\frac{p q}{p-q}}(|\ln t|) t^{-1} d t=\int_{|\ln b|}^{|\ln a|} \varphi_{v}^{\frac{p q}{p-q}}(y) \varphi_{g}^{\frac{p q}{p-q}}(y) d y, \\
& A_{1, k}^{\frac{p q}{p-q}} \leq \int_{a}^{b}\left(\int_{a}^{t} \varphi_{v}^{q}(|\ln x|) x^{q\left(-\beta_{v}+k\right)} d x\right)^{\frac{p}{p-q}} \\
& \times\left(\int_{t}^{b} \varphi_{g}^{p^{\prime}}(|\ln x|) x^{p^{\prime}\left(-\beta_{g}+r-k-1\right)} d x\right)^{\frac{p(q-1)}{p-q}} t^{p^{\prime}\left(-\beta_{g}+r-k-1\right)} \varphi_{g}^{p^{\prime}}(|\ln t|) d t \\
& \underset{p, q, r, \beta_{g}, c_{*}}{[5.17,[5.2} \int_{a}^{b} \varphi_{v}^{\frac{p q}{p-q}}(|\ln t|) t^{\frac{p q}{p-q}\left(-\beta_{v}+k+\frac{1}{q}\right)} \varphi_{g}^{\frac{p q}{p-q}}(|\ln t|) t^{\frac{p q}{p-q}\left(-\beta_{g}+r-k-1+\frac{1}{p^{\prime}}\right)-1} d t \\
& =\int_{a}^{b} \varphi_{v}^{\frac{p q}{p-q}}(|\ln t|) \varphi_{g}^{\frac{p q}{p-q}}(|\ln t|) t^{-1} d t=\int_{|\ln b|}^{|\ln a|} \varphi_{v}^{\frac{p q}{p-q}}(y) \varphi_{g}^{\frac{p q}{p-q}}(y) d y .
\end{aligned}
$$

Proof of Proposition 4. Whenever $\ln \frac{b}{a} \leq 1$, the claim follows from Proposition 2, Consider the case where $\ln \frac{b}{a}>1$.

Let $\beta_{v}<\frac{1}{q}$. 
We estimate $B_{0}$ and $B_{1}$ from above:

$$
\begin{aligned}
& B_{0}=\sup _{t \in(a, b)}\left(\int_{a}^{t} x^{-\beta_{v} q} \varphi_{v}^{q}(|\ln x|)(t-x)^{q(r-1)} d x\right)^{\frac{1}{q}}\left(\int_{t}^{b} x^{-p^{\prime} \beta_{g}} \varphi_{g}^{p^{\prime}}(|\ln x|) d x\right)^{\frac{1}{p^{\prime}}} \\
& \text { 5.1, 5.2 } \\
& \underset{p, q, r, \beta_{g}, c_{*}}{\stackrel{15.1}{15.2}} \sup _{t \in(a, b)}(t-a)^{r-1} \varphi_{v}(|\ln t|) t^{\frac{1}{q}-\beta_{v}} \varphi_{g}(|\ln t|) t^{\frac{1}{p^{\prime}}-\beta_{g}} \leq\left\|\varphi_{g} \varphi_{v}\right\|_{L_{\infty}[|\ln b|,|\ln a|]}, \\
& B_{1}=\sup _{t \in(a, b)}\left(\int_{a}^{t} x^{-\beta_{v} q} \varphi_{v}^{q}(|\ln x|) d x\right)^{\frac{1}{q}}\left(\int_{t}^{b}(x-t)^{p^{\prime}(r-1)} x^{-p^{\prime} \beta_{g}} \varphi_{g}^{p^{\prime}}(|\ln x|) d x\right)^{\frac{1}{p^{\prime}}}
\end{aligned}
$$

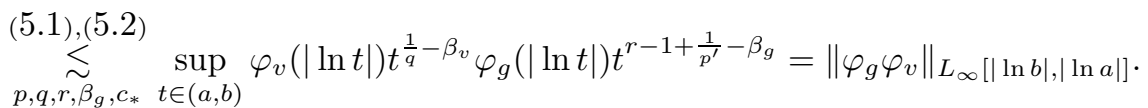

The case where $\beta_{v}>r-1+\frac{1}{q}$ is treated in a similar way.

Let $k-1+\frac{1}{q}<\beta_{v}<k+\frac{1}{q}, k \in\{1, \ldots, r-1\}$. Then

$$
\begin{aligned}
& \left\|\widetilde{I}_{r, g, v}^{a, b, k}\right\|_{L_{p}[a, b] \rightarrow L_{q}[a, b]} \underset{p, q, r}{\lesssim} B_{0, k}+B_{1, k}, \\
& B_{0, k}=\sup _{t \in(a, b)}\left(\int_{t}^{b} \varphi_{v}^{q}(|\ln x|) x^{-\beta_{v} q}(x-a)^{q(k-1)} d x\right)^{1 / q} \\
& \times\left(\int_{a}^{t} \varphi_{g}^{p^{\prime}}(|\ln x|) x^{-\beta_{g} p^{\prime}}(x-a)^{p^{\prime}(r-k)} d x\right)^{1 / p^{\prime}} \\
& \leq \sup _{t \in(a, b)}\left(\int_{t}^{b} x^{q\left(-\beta_{v}+k-1\right)} \varphi_{v}^{q}(|\ln x|) d x\right)^{1 / q}\left(\int_{a}^{t} x^{p^{\prime}\left(-\beta_{g}+r-k\right)} \varphi_{g}^{p^{\prime}}(|\ln x|) d x\right)^{1 / p^{\prime}} \\
& \text { 5.1, 5.2 } \\
& \underset{p, q, r, \beta_{g}, c_{*}}{\lesssim} \sup _{t \in(a, b)} \varphi_{v}(|\ln t|) t^{-\beta_{v}+k-1+\frac{1}{q}} \varphi_{g}(|\ln t|) t^{-\beta_{g}+r-k+\frac{1}{p^{\prime}}} \\
& =\left\|\varphi_{g} \varphi_{v}\right\|_{L_{\infty}[|\ln b|,|\ln a|]}, \\
& B_{1, k}=\sup _{t \in(a, b)}\left(\int_{a}^{t} \varphi_{v}^{q}(|\ln x|) x^{-\beta_{v} q}(x-a)^{q k} d x\right)^{1 / q} \\
& \times\left(\int_{t}^{b} \varphi_{g}^{p^{\prime}}(|\ln x|) x^{-\beta_{g} p^{\prime}}(x-a)^{p^{\prime}(r-k-1)} d x\right)^{1 / p^{\prime}} \\
& 5.1,55.2 \\
& \begin{array}{l}
\underset{p, q, r, \beta_{g}, c_{*}}{\stackrel{5.1}{, 5.2}} \sup _{t \in(a, b)} \varphi_{v}(|\ln t|) t^{-\beta_{v}+k+\frac{1}{q}} \varphi_{g}(|\ln t|) t^{-\beta_{g}+r-k-1+\frac{1}{p^{\prime}}} \\
=\left\|\varphi_{g} \varphi_{v}\right\|_{\left.L_{\infty}[|\ln b|, \mid \ln a]\right] .}
\end{array}
\end{aligned}
$$

In conclusion, the author expresses her sincere gratitude to I. G. Tsar'kov for suggesting the problem, to V. D. Stepanov and D. D. Haroske for references, and to A. S. Kochurov for reading the manuscript.

\section{REFERENCES}

[1] S. Heinrich, On the relation between linear n-widths and approximation numbers, J. Approx. Theory 58 (1989), no. 3, 315-333. MR1012680 (90g:41036)

[2] V. M. Tikhomirov, Diameters of sets in functional spaces and the theory of best approximations, Uspekhi Mat. Nauk 15 (1960), no. 3, 81-120; English transl., Russian Math. Surveys 15 (1960), no. $3,75-111$. MR0117489 (22:8268)

[3] S. B. Babadzhanov and V. M. Tikhomirov, Diameters of a function class in an $L^{p},(p>1)$, Izv. Akad. Nauk Uz.SSR Ser. Fiz.-Mat. Nauk 1967, no. 2, 24-30. (Russian) MR0209747 (35:643) 
[4] A. P. Buslaev and V. M. Tikhomirov, The spectra of nonlinear differential equations and widths of Sobolev classes, Mat. Sb. 181 (1990), no. 12, 1587-1606; English transl., Math. USSR-Sb. 71 (1992), no. 2, 427-446. MR1099516 (92f:46036)

[5] R. S. Ismagilov, Diameters of sets in normed linear spaces, and the approximation of functions by trigonometric polynomials, Uspekhi Mat. Nauk 29 (1974), no. 3, 161-178; English transl., Russian Math. Surveys 29 (1974), no. 3, 169-186. MR0407509 (53:11284)

[6] B. S. Kashin, The widths of certain finite-dimensional sets and classes of smooth functions, Izv. Akad. Nauk SSSR Ser. Mat. 41 (1977), no. 2, 334-351; English transl., Math. USSR-Izv. 11 (1977), no. 2, 317-333. MR0481792 (58:1891)

[7] A. Pietsch, s-numbers of operators in Banach spaces, Studia Math. 51 (1974), 201-223. MR0361883 $(50: 14325)$

[8] M. I. Stesin, Aleksandrov diameters of finite-dimensional sets and of classes of smooth functions, Dokl. Akad. Nauk SSSR 220 (1975), no. 6, 1278-1281; English transl., Soviet Math. Dokl. 16 (1975), no. 1, 252-256. MR0617942 (58:29729)

[9] V. E. Maĭorov, Discretization of the problem of diameters, Uspekhi Mat. Nauk 30 (1975), no. 6, 179-180. (Russian) MR0402349 (53:6170)

[10] Yu. I. Makovoz, A certain method of obtaining lower estimates for diameters of sets in Banach spaces, Mat. Sb. (N.S.) 87 (129) (1972), no. 1, 136-142; English transl. in Math. USSR-Sb. 16 (1972). MR0291774(45:865)

[11] E. D. Gluskin, Norms of random matrices and diameters of finite-dimensional sets, Mat. Sb. (N.S.) 120 (162) (1983), no. 2, 180-189; English transl. in Math. USSR-Sb. 48 (1984). MR0687610 (84g:41021)

[12] V. M. Tikhomirov, Some questions in approximation theory, Moskov. Univ., Moscow, 1976. (Russian) MR0487161 (58:6822)

[13] _ Approximation theory, Itogi Nauki i Tekhniki Sovrem. Probl. Mat. Fundam. Naprav., vol. 14, VINITI, Moscow, 1987, pp. 103-260; English transl., Encyclopaedia Math. Sci., vol. 14, Springer-Verlag, Berlin, 1990, pp. 93-243. MR0915774 MR.1074378(91e:00001)

[14] A. Pinkus, $n$-widths in approximation theory, Ergeb. Math. Grenzgeb. (3), Bd. 7, Springer, Berlin, 1985. MR0774404 (86k:41001)

[15] M. A. Lifshits and W. Linde, Approximation and entropy numbers of Volterra operators with application to Brownian motion, Mem. Amer. Math. Soc. 157 (2002), no. 745. MR1895252 (2004g:47066)

[16] D. E. Edmunds and J. Lang, Approximation numbers and Kolmogorov widths of Hardy-type operators in a non-homogeneous case, Math. Nachr. 279 (2006), no. 7, 727-742. MR2226408 (2007b:47112)

[17] J. Lang, Improved estimates for the approximation numbers of Hardy-type operators, J. Approx. Theory 121 (2003), no. 1, 61-70. MR:1962996 (2003m:41036)

[18] E. N. Lomakina and V. D. Stepanov, On asymptotic behaviour of the approximation numbers and estimates of Schatten-von Neumann norms of the Hardy-type integral operators, Function Spaces and Applications (Delhi, 1997), Narosa, New Delhi, 2000, pp. 153-187. MR1974800 (2004b:47080)

[19] _ Asymptotic estimates for the approximation and entropy numbers of the one-weight Riemann-Liouville operator, Mat. Tr. 9 (2006), no. 1, 52-100. (Russian) MR 2251331 (2997k:47077)

[20] A. A. Vasil'eva, Estimates of widths for weighted Sobolev classes, Mat. Sb. 201 (2010), no. 7, 15-52; English transl., Sb. Math. 201 (2010), no. 7-8, 947-984. MR2907813

[21] _ Criterion for the existence of a continuous embedding of a weighted Sobolev class on a closed interval and on a semiaxis, Russ. J. Math. Phys. 16 (2009), no. 4, 543-562. MR2587811 $(2011 \mathrm{j}: 46057)$

[22] Ė. N. Batuev and V. D. Stepanov, Weighted inqualities of Hardy type, Sibirsk. Mat. Zh. 30 (1989), no. 1, 13-22; English transl., Siberian Math. J. 30 (1989), no. 1, 8-16. MR0995015 (90f:26017)

[23] V. D. Stepanov, Two-weighted estimates for Riemann-Liouville integrals, Izv. Akad. Nauk SSSR Ser. Mat. 54 (1990), no. 3, 645-656; English transl., Math. USSR-Izv. 36 (1991), no. 3, 669-681. MR:1072699(92b:44006)

[24] _ Two-weighted estimates for Riemann-Liouville integrals, Rep. no. 39, Ceskoslov. Akad. Věd. Mat. Ústav., Prague, 1988, pp. 1-28.

[25] — Weighted norm inequalities for integral operators and related topics, Nonlinear Analysis, Function Spaces and Applications, Vol. 5 (Prague, 1994), Prometheus, Prague, 1994, pp. 139-175. MR $1322312(96 \mathrm{~m}: 26019)$

[26] Weighted norm inequalities of Hardy type for a class of integral operators, J. London Math. Soc. (2) 50 (1994), no. 1, 105-120. MR1277757(95h:47047) 
[27] A. Kufner and G. P. Kheınig, The Hardy inequality for higher-order derivatives, Trudy Mat. Inst. Steklova 192 (1990), 105-113; English transl., Proc. Steklov Inst. Math. 1992, no. 3, 113-121. MR $1097892(92 \mathrm{e}: 26008)$

[28] D. D. Haroske and L. Skrzypczak, Entropy and approximation numbers of embeddings of function spaces with Muckenhoupt weights. I, Rev. Mat. Complut. 21 (2008), no. 1, 135-177. MR2408040 (2009g:46060)

[29] Entropy numbers of embeddings of function spaces with Muckenhoupt weights. III. Some limiting cases, J. Funct. Spaces Appl. 9 (2011), no. 2, 129-178. MR2827745 (2012e:46070)

[30] G. Pisier, The volume of convex bodies and Banach space geometry, Cambridge Tracts in Math., vol. 94, Cambridge Univ. Press, Cambridge, 1989. MR.1036275 (91d:52005)

Lomonosov Moscow State University, GSP-1, Leninskie Gory, Moscow 119991, Russia

E-mail address: vasilyeva_nastya@inbox.ru

Received 15/JUN/2010

Translated by THE AUTHOR 